CIRJE-F-1013

\title{
Derivatives Pricing with Market Impact and Limit Order Book
}

\author{
Taiga Saito \\ The University of Tokyo \\ Akihiko Takahashi \\ The University of Tokyo
}

May 2016; Revised in August 2017

CIRJE Discussion Papers can be downloaded without charge from:

http://www.cirje.e.u-tokyo.ac.jp/research/03research02dp.html

Discussion Papers are a series of manuscripts in their draft form. They are not intended for circulation or distribution except as indicated by the author. For that reason Discussion Papers may not be reproduced or distributed without the written consent of the author. 


\title{
Derivatives Pricing with Market Impact and Limit Order Book
}

\author{
Taiga Saito $^{\text {a }}$, Akihiko Takahashi ${ }^{\mathrm{b}}$, \\ ${ }^{a}$ Graduate School of Economics, The University of Tokyo \\ 7-3-1 Hongo, Bunkyo-ku, Tokyo 113-0033, Japan \\ ${ }^{\mathrm{b}}$ Graduate School of Economics, The University of Tokyo \\ 7-3-1 Hongo, Bunkyo-ku, Tokyo 113-0033, Japan
}

\begin{abstract}
This paper investigates derivatives pricing under existence of liquidity costs and market impact for the underlying asset in continuous time. Firstly, we formulate the charge for the liquidity costs and the market impact on the derivatives prices through a stochastic control problem that aims to maximize the mark-to-market value of the portfolio less the quadratic variation multiplied by a risk aversion parameter during the hedging period and the liquidation cost at maturity. Then, we obtain the derivatives price by reduction of this charge from the premium in the Bachelier model. Secondly, we consider a second order semilinear partial differential equation (PDE) of parabolic type associated with the control problem, which is analytically solved or approximated by an asymptotic expansion around a solution to an explicitly solvable nonlinear PDE. Finally, we present numerical examples of the pricing for a variance option and a European call option, and show comparative static analyses.
\end{abstract}

Key words: Stochastic control; Application in finance; Derivatives pricing.

\section{Introduction}

In this paper, we consider derivatives pricing under the existence of the market impact and the liquidity costs on the underlying asset price, which are caused by the transactions of the hedger. After formulating the hedging cost through a stochastic control problem, which is a generalized form of a linear-quadratic control problem, we provide a scheme to compute the cost which is solved analytically or approximated by an asymptotic expansion of a second order semilinear PDE of parabolic type. This asymptotic expansion is novel in that the solution is expanded around that of an explicitly solvable semilinear PDE. This is different from previous works on asymptotic expansions for derivatives pricing (see Takahashi [16] and the references therein), which typically make expansion around linear PDEs.

Estimation of the total liquidity cost during the hedging period is the most essential factor in pricing in practice, since banks may have losses by the price spreads which they pay in every hedging transaction. Prediction of the effect of market impact on the hedging cost is also important, especially when banks trade derivatives on illiq-

\footnotetext{
* Forthcoming in "Automatica".

${ }^{\star \star}$ All the contents expressed in this research are solely those of the authors and do not represent any views or opinions of any institutions. The authors are not responsible or liable in any manner for any losses and/or damages caused by the use of any contents in this research. This research is supported by CARF (Center for Advanced Research in Finance). This research is supported by JSPS KAKENHI Grant Number 25380389.
}

Email addresses: saitotaiga@hotmail.com (Taiga Saito), akihikot@e.u-tokyo.ac.jp (Akihiko Takahashi). uid underlying assets such as low liquidity stocks and illiquid foreign exchange rates. Moreover, when banks quote a derivatives price in biddings, the estimation of these costs is occasionally the only differentiator among the participants. Despite these facts, the estimation of the entire hedging cost is usually done by traders' rules of thumb. This study provides a quantitative method to estimate the cost. In addition, our model incorporates the liquidation cost at maturity for the two settlement types, physical and cash settlements, and slippages on execution volume of the underlying asset, which are observed in trading of illiquid assets in practice.

As for related literatures, Li and Almgren [11] deal with hedging an option under the existence of liquidity costs and market impact. Guéant and $\mathrm{Pu}$ [7] consider indifference pricing of a hedger with an exponential utility on the mark-to-market value of the hedging portfolio at maturity. After deriving the HJB equation for the optimization problem, Guéant and $\mathrm{Pu}$ [7] solve the HJB equation numerically by finding a maximum point at every grid of the discretized equation.

On the other hand, we adopt a different objective function from the one in Guéant and $\mathrm{Pu}$ [7] for the maximization. By assuming the mark-to-market value of the hedging portfolio at maturity less the terminal liquidation cost and the quadratic hedging error as the objective function to be maximized, the problem becomes a generalized form of the linear-quadratic control problem, where the related HJB equation reduces to a second order semilinear PDE of parabolic type. Then, depending on the payoff of derivatives, we analytically solve the semilinear PDE or asymptotically expand the solution of the PDE up to the first order. In detail, we expand the solution around that of a solvable semilinear PDE. The 
zeroth order part of the solution has a quadratic expression with respect to a state variable, whose coefficients satisfy an ODE or a linear PDE, and the first order part is a solution of a second order linear PDE. We solve the system of the ODE and PDEs through stochastic representations of the solutions by Feynman-Kac formula.

We note that Li and Almgren [11] only deal with intraday hedging on a specific date far away from the maturity, and hence does not consider pricing options, which is generalized in our study to hedging and pricing derivatives for the entire trading period. In particular, we remove the crucial assumption in Li and Almgren [11] that the derivatives gamma is constant. This assumption is only applicable to intraday trading of a specific trading date far from the maturity. Note that Li and Almgren [11] deal with the general gamma only in a no market impact case. When the underlying asset price is staying in the at the money area and the trading date is near the maturity, calculation of the optimal hedging strategy is particularly important since the hedging is difficult due to large derivatives gamma for European call or put options. Further, in contrast to Li and Almgren [11], the liquidation cost at maturity for the two settlement types, physical and cash settlements, and slippages on execution volume of the underlying asset are also incorporated in our model.

We remark that the derivatives gamma is the second order differential of the value of the derivatives with respect to the underlying asset price. The delta, the first order differential of the value of the derivatives with respect to the underlying asset price, is the quantity of the underlying asset to offset in order to keep the mark-to-market value of the hedger's portfolio unaffected from underlying asset price movements in a short period. Thus, when the gamma is high, the hedger has to trade a large number of units for the underlying assets every time the underlying asset price moves. The gamma is particularly high when the trading date is near the maturity or the underlying asset price is staying in the area where the convexity of the derivatives payoff is large, such as at the money area of the European call or put option.

While Li and Almgren [11] do not show any numerical experiment and Guéant and $\mathrm{Pu}[7]$ provide only one example with market impact, our study presents various cases of derivatives prices under the existence of market impact. We provide derivatives prices for a variance option in physical settlement, which are analytically solvable, and those for a European call option in physical settlement, which are obtained through the asymptotic expansion. Note that the derivatives with a variance option is an important example corresponding to a variance contract that pays realized variance of the underlying asset price at maturity.

As for numerical methods for solving the HJB equations, Aliyu [1] investigates a transformation approach for solving the equation by reducing the equation into a set of coupled algebraic-differential inequalities. Huang et al. [9] propose a semi-meshless discretization where the spatial discretization is based on a collocation scheme using the global radial basis functions. Beard et al. [3] apply the Galerkin's approximation method to a solution of the generalized HJB equation in a deterministic control problem. Crespo and Sun [4] present a method for find- ing optimal controls by the generalized cell mapping and the short-time Gaussian approximation scheme.

This paper is organized as follows: Section 2 explains our model. Section 3 provides an asymptotic expansion of an associated semilinear PDE with its coefficients' computation in Section 4. Section 5 provides examples with numerical experiments in Section 6. Section 7 compares our derivatives price with that of Guéant and $\mathrm{Pu}$ [7]. Section 8 concludes.

\section{Model}

In this section, we introduce an optimal hedging problem for a derivatives hedger, who is the sole rational large investor in the market of the underlying asset, under the existence of liquidity costs and market impact.

Since estimation of costs related to illiquidity is essential in derivatives pricing in practice, we incorporate important factors on illiquidity (a finite variation process for the units of orders submitted by the hedger, temporary and permanent impacts on prices, execution slippages on the trade units, and the liquidation cost at maturity which depends on the settlement types) in modeling.

Firstly, we assume a finite variation process for units of orders submitted by the hedger in Section 2.1. This is different from the delta hedging in the Black-Sholes and the Bachelier model, since the delta hedging in these models can be done at any instant to offset the fluctuation of the derivatives price, and thus the underlying asset position has an infinite total variation, which is impossible in practice especially for illiquid markets.

Also, we take market frictions into consideration, i.e. slippages on the trade units, temporary and permanent impacts on the mid price, and the liquidity cost at maturity in Section 2.2.

Specifically, the hedger enters a long/short derivatives position and starts hedging the position with the underlying asset. The hedger mark-to-markets the portfolio with the Bachelier model for the derivatives position and with the mid price for the underlying asset position. At inception of the trading, the hedger exchanges the initial delta units of the underlying asset based on the Bachelier model at mid price with the counterparty of the derivatives. We explain these points in detail in the following subsections.

\subsection{Order volume and asset price processes}

The hedger aims to maximize his/her expected utility which is risk neutral or risk averse. The hedger holds the derivatives position at inception of the trading by paying $m_{0}$ as the premium.

Let $[0, T]$ be the trading period, where 0 is the initial time of the trading and $T$ is the maturity of derivatives. Let $\left(\Omega, \mathcal{F},\left\{\mathcal{F}_{t}\right\}_{0 \leq t \leq T}, \mathbf{P}\right)$ be the filtered probability space satisfying the usual conditions. We consider an economy that consists of a money market account and an underlying asset. We assume that the risk-free interest rate is zero, which implies that the price of the money market account is always 1 . Let $\theta_{t}$ be a $\left\{\mathcal{F}_{t}\right\}$-adapted process which satisfies $\mathbf{E}\left[\int_{0}^{T} \theta_{s}^{2} d s\right]<\infty,\left(W_{t}, W_{t}^{\perp}\right)$ be a two dimensional $\left\{\mathcal{F}_{t}\right\}$-Brownian Motion, $\sigma$ and $\delta$ be positive constants, and $\epsilon$ be nonnegative. Let $X_{t}$ be the number of units of the submitted orders by the hedger to market makers by time $t$, which is differentiable with 
derivative $\theta_{t}$, that is, $X_{t}=\int_{0}^{t} \theta_{s} d s$. In other words, $\theta_{t}$ is the number of units of orders for the underlying asset that the hedger submits to the market makers per period, or the speed of order placement for the underlying asset for hedging. (buy orders (when the sign is positive) or sell orders (when the sign is negative)).

The reason why we assume absolute continuity with respect to time for the accumulated order volume from the hedger is that in the real world, the order volume has a finite total variation, which corresponds to the integrability of $\theta, \int_{0}^{T}\left|\theta_{s}\right| d s<\infty$. If $X$ includes a term of a stochastic integration with respect to a Brownian motion, the total variation of the hedger's position can be infinite in the finite interval. This corresponds to an infinite order submission volume, which is impossible in the real word. Hence, the absolute continuity of the underlying asset position is particularly important in trading of an illiquid asset as in Longstaff [12], for example.

We define the mid price process of the underlying asset $P_{t}$ as

$$
P_{t}=P_{0}+\sigma W_{t}+\epsilon X_{t}, 0 \leq t \leq T .
$$

This indicates that the permanent market impact on the mid price process $\epsilon X_{t}$ is proportional to $X_{t}$, the number of units of the orders submitted by the hedger until time $t$. This means that if the hedger, the sole large trader, places a large number of buying (selling) orders, then there is a positive (negative) value of the permanent impact on the mid price. It is also known that the this formulation of market impact does not admit an arbitrage with round trip strategy (e.g. Gatharel [6] ). $\sigma W_{t}$ is the aggregate random effect on the price by the rest of market participants' trading, which is naturally modeled as a martingale. More elaborate modeling of agents other than a large investor will be one of our future research topics.

Remark 1 Note that this expression of the mid price process with the Gaussian random variable $\sigma W_{t}$ and the permanent market impact as in (1) is consistent with the literature of optimal execution with market impact (e.g. See Almgren and Chriss [2] and Gatheral [6]). Moreover, Proposition 2 in Schachermayer and Teichmann [15] shows that Gaussian models well approximate lognormal models with short maturities and at-the-money strike, which are most important cases in our analysis, since the market impact and the liquidity costs are particularly large due to the high derivatives gamma and the changes in the delta. For example, with $0.2 \%$ and 0.5 years used in our examples in Section 6, it implies that the errors are less than $0.17 \%$ in price and $0.03 \%$ in implied volatility, which are small enough compared to the size of the costs related to the illiquidity. In addition, we can naturally consider interest rate options and spread options, whose underlying asset prices may be negative.

Remark 2 However, our technique has a limitation in that a standard (Black-Scholes-Merton type) lognormalbased model itself does not satisfy mathematical conditions necessary for the proposed expansion method. One possible way to deal with derivatives under a lognormal model is to approximate the underlying asset price by the following quadratic function based on the Taylor expansion up to the second order around a given $x_{0}$ :

$$
P_{T}=e^{x} \sim e^{x_{0}}\left(1+\left(x-x_{0}\right)+\frac{1}{2}\left(x-x_{0}\right)^{2}\right) .
$$

Then, for example, by taking $x_{0}=\log K$, we have the second order approximation of the call payoff as

$$
\left(e^{x}-K\right)^{+} \sim K\left(\frac{1}{2}(x-\log K)^{2}+(x-\log K)\right)^{+}
$$

where $x=\log P_{0}+\left(-\frac{1}{2} \sigma^{2} T\right)+\sigma W_{T}+\epsilon X_{T}$. This approximated payoff satisfies the mathematical conditions for our expansion scheme. Pricing derivatives under the lognormal assumption will be a topic for our future research.

\subsection{Execution slippages on price and order volume}

We consider the following execution slippages on the price and the order volume of the hedger.

Firstly, as for the slippage on the price, let $\eta$ be a nonnegative constant and $\tilde{P}\left(\theta_{t}\right)$ be the execution price when the hedger submits $\theta_{t} d t$ units of orders for the underlying asset during the period from $t$ to $t+d t$.

We define $\tilde{P}\left(\theta_{t}\right)$ as

$$
\tilde{P}\left(\theta_{t}\right)=P_{t}+\eta \theta_{t}
$$

(2) indicates that the temporary price impact (the execution slippage on price) $\eta \theta_{t}$, which is added on (for buy orders) or reduced from (for sell orders) the mid price $P_{t}$, is proportional to $\theta_{t}$. This means that when the hedger submits a larger number of units of buying (selling) orders per period to the market makers, he/she receives a higher (lower) price for the buying (selling) order execution.

This formulation of the temporary impact (the execution slippage on price) also corresponds to trading with a limit order book in $[t, t+d t]$ where $\frac{1}{2 \eta} d t$ units of the limit orders are placed per price. If the market makers fill an order to buy $\theta_{t} d t$ units of the underlying asset, they buy at the prices from $P_{t}$ to $P_{t}+2 \eta \theta_{t}\left(=P_{t}+\frac{\theta_{t} d t}{\frac{1}{2 \eta} d t}\right)$. This implies that they buy $\theta_{t} d t$ units of the underlying asset at the average price of $P_{t}+\eta \theta_{t}$, which is $\tilde{P}_{t}$. Conversely, if the market makers fill an order to sell $\left|\theta_{t}\right| d t$ units of the underlying asset, they sell at the price from $P_{t}$ to $P_{t}-2 \eta\left|\theta_{t}\right|$, which means that the market makers sell $\left|\theta_{t}\right| d t$ units of the underlying asset at the average price of $P_{t}-\eta\left|\theta_{t}\right|=P_{t}+\eta \theta_{t}=\tilde{P}_{t}$.

(For a general relation between the limit order book density and the price spread, see Saito [14] for instance.)

This type of formulation of the temporary execution slippage and the permanent market impact as in (1) and (2) is also observed in the literature of optimal execution, Almgren and Chriss [2] and Rogers and Singh [13] for example.

Before we introduce the execution slippage on the order volume for the hedger, we define the derivatives gamma in the Bachelier model which corresponds to the model substituting $\epsilon=0$ in (1) and $\eta=0$ in (2). Let $h: \mathbf{R} \rightarrow \mathbf{R}$ be the derivatives payoff function, which satisfies a polynomial growth condition and represents the derivatives payoff at $T$ as a function of the mid price of the underlying asset $x_{1}$. Let $g:[0, T] \times \mathbf{R} \rightarrow \mathbf{R}$ be the unique solution of the PDE

$$
g_{t}+\frac{1}{2} \sigma^{2} g_{x_{1} x_{1}}=0, g\left(T, x_{1}\right)=h\left(x_{1}\right),
$$

satisfying a polynomial growth condition: there exist positive constants $C$ and $k$ such that $\left|g\left(t, x_{1}\right)\right| \leq C(1+$ $\left.\left|x_{1}\right|^{k}\right), 0 \leq \forall t \leq T, \forall x_{1} \in \mathbf{R}$. 
Here $\frac{\partial}{\partial t} g$ and $\frac{\partial^{2}}{\partial x_{1}^{2}} g$ are denoted by $g_{t}$ and $g_{x_{1}, x_{1}}$, respectively. Hereafter, we use these notations with subscripts for partial derivatives. Note that $g$ is given by $g\left(t, x_{1}\right)=\int_{\mathbf{R}} \frac{1}{\sqrt{2 \pi \sigma^{2}(T-t)}} \exp \left(-\frac{\left(x_{1}-\xi\right)^{2}}{2 \sigma^{2}(T-t)}\right) h(\xi) d \xi$,

$0 \leq \forall t<T, \forall x_{1} \in \mathbf{R}$. (For instance, see Remark 5.7.8 in Karatzas and Shreve [10]. Hereafter, the number before the dot in the reference of theorems indicates the chapter of the book.)

We denote $g_{x_{1} x_{1}}\left(t, x_{1}\right)$ by $\Gamma\left(t, x_{1}\right)$, which is the derivatives gamma in the Bachelier model. We also note that in the Bachelier model, when the absolute value of the derivatives gamma is high, the delta position of the derivatives $g_{x_{1}}\left(t, x_{1}\right)$ changes frequently as the underlying asset price moves. In such a case, the derivatives hedger has to rebalance his/her underlying asset position accordingly in order to avoid change in the mark-to-market of the portfolio.

Then, we introduce the execution slippage on the order volume for the hedger as follows. The underlying asset position starts with $\tilde{X}_{0}$, which can be the delta amount exchanged with the counterparty of the derivatives for example. The delta exchange with the counterparty is done off-market at the mid price $P_{0}$ (i.e. with value $\tilde{X}_{0} P_{0}$, see (9)) between the two counterparties whose initial hedging volumes are the same in absolute value but have the opposite sign. Let $\tilde{X}_{t}$ be the position of the underlying asset of the hedger, which is a $\left\{\mathcal{F}_{t}\right\}$-adapted process satisfying

$$
\tilde{X}_{t}=\tilde{X}_{0}+X_{t}+\delta \int_{0}^{t} \sigma \Gamma\left(t, P_{t}\right) d W_{t}^{\perp}
$$

We interpret this as follows. $\delta \int_{0}^{t} \sigma \Gamma\left(t, P_{t}\right) d W_{t}^{\perp}$ is the execution slippage, the mismatch volume which arises when the market markers clear the orders from the hedger via the limit order book.

We remark that the setting is an incomplete market due to the existence of $d W_{t}^{\perp}$ in the hedging position, which is independent of $d W_{t}$, a driver of the mid price process. Moreover, the market is not perfectly complete even when $\delta=0$ since the hedging strategy is assumed to be absolutely continuous with respect to time and does not contain $d W_{t}$, the Brownian motion term. In addition, there is a market friction because of the execution slippages on the price and volume. We further put the next assumption that is necessary for asymptotic expansions in the following sections.

Assumption 1 There exist positive constants $c_{1}$ and $c_{2}$ such that

$$
c_{1} \leq\left|\Gamma\left(t, x_{1}\right)\right| \leq c_{2}, \forall t \in[0, T], \forall x_{1} \in \mathbf{R} .
$$

Moreover, $\Gamma\left(t, x_{1}\right)$ is of class $C^{1,2}([0, T] \times \mathbf{R})$ and $\Gamma_{x_{1}}\left(t, x_{1}\right)$ is bounded on $[0, T] \times \mathbf{R}$.

Then, let us introduce Case 1 (variance option) and Case 2(smooth modification of European call option), which satisfy this assumption. Numerical examples for the derivatives payoff corresponding to Case 1 and Case 2 will be presented in Example 1 and Example 2 in Section 5, respectively.

Case 1 (Variance option). For $K>0$ and $\gamma \neq 0$, $h\left(x_{1}\right)=\frac{\gamma}{2}\left(x_{1}-K\right)^{2}$ satisfies Assumption 1. In fact, by Feynman-Kac Theorem (e.g. Theorem 4.4.2 in Karatzas and Shreve [10] $), g\left(t, x_{1}\right)=\mathbf{E}\left[h\left(x_{1}+\sigma W_{T-t}\right)\right]=$ $\frac{\gamma}{2}\left(\left(x_{1}-K\right)^{2}+\sigma^{2} T\right), g_{x_{1}}\left(t, x_{1}\right)=\gamma\left(x_{1}-K\right), \Gamma\left(t, x_{1}\right)=$ $\gamma, \Gamma_{x_{1}}\left(t, x_{1}\right)=0$.

Case 2 (Smooth modification of European call option). For $l, \bar{\delta}>0$ and $0<c_{1}<\frac{1}{2 l}$, let $h\left(x_{1}\right)=\frac{l}{4}+\int_{K}^{x_{1}}\left(\frac{1}{2}+\right.$ $\left.\int_{K}^{v} h^{\prime \prime}(s) d s\right) d v$ where $h^{\prime \prime}\left(x_{1}\right)=c_{1}+\left(\frac{1}{2 l}-c_{1}\right) g_{l, \bar{\delta}}\left(x_{1}-K\right)$. Here, $g_{l, \bar{\delta}}\left(x_{1}\right)$ is a function of class $C^{\infty}(\mathbf{R})$ satisfying $g_{l, \bar{\delta}}\left(x_{1}\right)=\left\{\begin{array}{l}1, \text { if }-l<x_{1}<l, \\ 0, \text { if } x_{1}<-l-\bar{\delta}, l+\bar{\delta}<x_{1},\end{array}\right.$ $0 \leq g_{l, \bar{\delta}}\left(x_{1}\right) \leq 1$ (if $\left.-l-\bar{\delta} \leq x_{1} \leq-l, l \leq x_{1} \leq l+\bar{\delta}\right)$. In detail, we can take such $g_{l, \bar{\delta}}\left(x_{1}\right)$ that satisfies (2.2) as $g_{l, \bar{\delta}}\left(x_{1}\right)=\left\{\begin{array}{l}\frac{\phi\left(x_{1}-(-l-\bar{\delta})\right)}{\phi\left(x_{1}-(-l-\delta)\right)+\phi\left(-l-x_{1}\right)}, \text { if }-l-\bar{\delta} \leq x_{1} \leq-l \\ \frac{\phi\left(l+\bar{\delta}-x_{1}\right)}{\phi\left(l+\bar{\delta}-x_{1}\right)+\phi\left(x_{1}-l\right)}, \text { if } l \leq x_{1} \leq l+\bar{\delta},\end{array}\right.$ where $\phi\left(x_{1}\right)=\exp \left(-\frac{1}{x_{1}}\right)$ (if $x_{1} \geq 0$ ), 0 (otherwise). Then, $h\left(x_{1}\right)$ satisfies Assumption 1 . Note that $h\left(x_{1}\right)$ is interpreted as an approximation of the call option payoff $\left(x_{1}-K\right)^{+}$, since if we take limit as $l, \bar{\delta}, c_{1} \downarrow 0, h\left(x_{1}\right)$ converges to $\left(x_{1}-K\right)^{+}$.

\subsection{Settlement types and liquidation cost at maturity}

We consider two types of derivatives settlements at maturity, the cash and the physical settlement. In cash settlement, the seller pays the derivatives payoff in cash to the buyer. For example, for a European call option whose strike price is $K$, if the mid underlying asset price at maturity is $P_{T},\left(P_{T}-K\right)^{+}$is paid in cash.

On the other hand, in physical settlement, there is a physical delivery of the underlying asset between the two counterparties. The delta amount of the underlying asset at maturity is delivered, that is, $g_{x_{1}}\left(T, P_{T}\right)$ units of the underlying asset is delivered from the seller to the buyer and $g_{x_{1}}\left(T, P_{T}\right) P_{T}-g\left(T, P_{T}\right)$ is paid in cash from the buyer to the seller. In particular, if $P_{T} \geq K$ for the European call option, one unit of the underlying asset is delivered from the seller to the buyer and the buyer pays $K$ in cash. If $P_{T}<K$, nothing happens.

Let $Y_{t}$ be the difference between $\tilde{X}_{t}$, the underlying asset position, and $\left(-g_{x_{1}}\left(t, P_{t}\right)\right)$, the delta amount to hold against the derivatives position in the Bachelier model:

$$
Y_{t}=\tilde{X}_{t}-\left(-g_{x_{1}}\left(t, P_{t}\right)\right)
$$

The hedger has to liquidate unnecessary volume of the underlying asset at maturity depending on the settlement type. In cash settlement, the hedger liquidates the whole underlying asset position $\tilde{X}_{T}$. In physical settlement, the hedger liquidates $Y_{T}=\tilde{X}_{T}-\left(-g_{x_{1}}\left(T, P_{T}\right)\right)$ of the underlying asset.

We suppose that there exists a third party who undertakes the liquidation volume from the hedger immediately with $b_{2}>0$ as the cost of liquidation per unit of the underlying asset. That is, the price spread (from the mid price $P_{T}$ at $T$ ) for the liquidation volume $\alpha$ is $b_{2} \alpha$, and the liquidation cost is $b_{2} \alpha^{2}\left(=\left(b_{2} \alpha\right) * \alpha\right)$. For instance, $\alpha=\tilde{X}_{T}$ for cash settlement, and $\alpha=Y_{T}$ for physical settlement. By the same argument as in the previous subsection, this price spread corresponds to the limit order book with a uniform density $\frac{1}{2 b_{2}}$. Namely, the following equations hold. $\int_{P_{T}}^{P_{T}+2 b_{2} \alpha} \frac{1}{2 b_{2}} d z=$ $\alpha, \frac{1}{\alpha} \int_{P_{T}}^{P_{T}+2 b_{2} \alpha} z \frac{1}{2 b_{2}} d z=P_{T}+b_{2} \alpha$.

Let $\psi: \mathbf{R}^{2} \rightarrow \mathbf{R}$ be a penalty function that represents the liquidation cost of the underlying asset at maturity as a function of $P_{T}$ and $Y_{T}$. For example, in the case 
of cash settlement, since the liquidation cost for $\tilde{X}_{T}$ of the underlying asset is $b_{2} \tilde{X}_{T}^{2}=b_{2}\left(Y_{T}-g_{x_{1}}\left(T, P_{T}\right)\right)^{2}=$ $b_{2}\left(Y_{T}^{2}-2 g_{x_{1}}\left(T, P_{T}\right) Y_{T}+g_{x_{1}}\left(T, P_{T}\right)^{2}\right), \psi$ is specified as $\psi\left(x_{1}, x_{2}\right)=b_{2}\left(x_{2}^{2}-2 g_{x_{1}}\left(T, x_{1}\right) x_{2}+g_{x_{1}}\left(T, x_{1}\right)^{2}\right), b_{2}>0$.

Similarly, in the case of physical settlement, since the liquidation cost for $Y_{T}$ of the underlying asset is $b_{2} Y_{T}^{2}$, $\psi$ becomes

$$
\psi\left(x_{1}, x_{2}\right)=b_{2} x_{2}^{2}, b_{2}>0 \text {. }
$$

\subsection{Optimal trading problem for the hedger}

Next, we introduce the optimal hedging problem for the hedger. Let $R_{t}$ be the mark-to-market value of the hedger's portfolio at $t . R_{t}$ is defined as the sum of the Bachelier price of the derivatives, the mid value of the underlying asset position, and the cash position, that is,

$$
\begin{aligned}
& R_{t}=g\left(t, P_{t}\right)+\tilde{X}_{t} P_{t}-\left(m_{0}+\tilde{X}_{0} P_{0}+\int_{0}^{t} \tilde{P}\left(\theta_{s}\right) \theta_{s} d s\right) \\
& =g\left(t, P_{t}\right)+\tilde{X}_{t} P_{t}-m_{0}-\tilde{X}_{0} P_{0}-\int_{0}^{t} P_{s} \theta_{s} d s-\eta \int_{0}^{t} \theta_{s}^{2} d s .
\end{aligned}
$$

Here, $m_{0}$ and $\tilde{X}_{0} P_{0}$ represent the payment of the derivatives premium and the initial exchange value of the underlying asset, respectively. $\int_{0}^{t} \tilde{P}\left(\theta_{s}\right) \theta_{s} d s$ in the first line stands for the accumulated loss/gain from the trading of the underlying asset until time $t$.

First note that by (1),

$$
d P_{t}=\epsilon \theta_{t} d t+\sigma d W_{t} \text {. }
$$

Applying Ito's formula to (6) and (9) with (3), we have

$$
\begin{aligned}
& d Y_{t}=\theta_{t}\left(1+\epsilon \Gamma\left(t, P_{t}\right)\right) d t+\tilde{\sigma} \Gamma\left(t, P_{t}\right) d \tilde{W}_{t}, \\
& d R_{t}=Y_{t} d P_{t}+\delta P_{t} \sigma \Gamma\left(t, P_{t}\right) d W_{t}^{\perp}-\eta \theta_{t}^{2} d t,
\end{aligned}
$$

where $\tilde{\sigma}=\sqrt{1+\delta^{2}} \sigma, \tilde{W}_{t}=\frac{1}{\sqrt{1+\delta^{2}}}\left(W_{t}+\delta W_{t}^{\perp}\right)$.

Then, we formulate the optimal hedging problem for the hedger. Let $\lambda \geq 0$ be a risk aversion parameter of the hedger. We suppose that the hedger aims to maximize

$$
\mathbf{E}\left[R_{T}-\psi\left(P_{T}, Y_{T}\right)-\lambda\langle R\rangle_{T}\right],
$$

which is the expectation of the mark-to-market value $R_{T}$ less the liquidation cost at maturity $\psi\left(P_{T}, Y_{T}\right)$ and the quadratic variation multiplied by the risk aversion parameter, $\lambda\langle R\rangle_{T}$, by choosing an optimal speed of order placement $\theta_{t}(0 \leq t \leq T)$. Here, $\langle R\rangle_{T}$ is the quadratic variation of $R_{T}:\langle\bar{R}\rangle_{T}=$ $\int_{0}^{T}\left(\sigma^{2} Y_{s}^{2}+\delta^{2} \sigma^{2} P_{s}^{2} \Gamma^{2}\left(s, P_{s}\right)\right) d s$. When $\lambda=0$, the objective function (13) is the expectation of the cash remaining after the maturity, and the hedger is considered to be risk-neutral. When $\lambda>0$, the additional term $-\lambda\langle R\rangle_{T}$ in the expectation indicates that the hedger is risk averse and he/she does not like the fluctuation of the mark-to-market value of the portfolio.

Remark 3 By definition (e.g. Chapter 1.5. in Karatzas and Shreve [10]), $d\langle R\rangle_{t}$ is intuitively interpreted as $\left(d R_{t}\right)^{2}$ and $\mathbf{E}\left[\langle R\rangle_{T}\right]=\int_{0}^{T} \mathbf{E}\left[\left(d R_{t}\right)^{2}\right]$ is considered to be an accumulation of the expected instantaneous second order variation (i.e. instantaneous variance) of $R_{t}$. In our objective function $\mathbf{E}\left[R_{T}-\psi\left(P_{T}, Y_{T}\right)-\lambda\langle R\rangle_{T}\right]$, the hedger tries to keep $\mathbf{E}\left[\left(d R_{t}\right)^{2}\right]$ low, which measures a fluctuation of the mark-to-market value of his/her portfolio, at the same time maximizing the expected markto-market value of the portfolio less the liquidation cost at maturity.
Note that by (12), we have

$$
\begin{gathered}
\mathbf{E}\left[R_{T}-\psi\left(P_{T}, Y_{T}\right)-\lambda\langle R\rangle_{T}\right] \\
=-m_{0}+g\left(0, P_{0}\right)-\mathbf{E}\left[\int_{0}^{T} L\left(s, P_{s}, Y_{s}, \theta_{s}\right) d s+\psi\left(P_{T}, Y_{T}\right)\right],
\end{gathered}
$$

where

$L\left(t, x_{1}, x_{2}, \theta\right)=\eta \theta^{2}-\epsilon x_{2} \theta+\lambda\left(\sigma^{2} x_{2}^{2}+\delta^{2} \sigma^{2} x_{1}^{2} \Gamma\left(t, x_{1}\right)^{2}\right)$.

Note that the highest premium $m_{0}$ such that the objective function is nonnegative becomes

$$
g\left(0, P_{0}\right)-\mathbf{E}\left[\int_{0}^{T} L\left(s, P_{s}, Y_{s}, \theta_{s}\right) d s+\psi\left(P_{T}, Y_{T}\right)\right],
$$

where $g\left(0, P_{0}\right)$ is the Bachelier price of the derivatives. Hence, $\mathbf{E}\left[\int_{0}^{T} L\left(s, P_{s}, Y_{s}, \theta_{s}\right) d s+\psi\left(P_{T}, Y_{T}\right)\right]$ can be interpreted as the hedging cost of the derivatives under the existence of the market impact.

$$
\begin{aligned}
& \text { Let } f\left(t, x_{1}, \theta\right)=\left(\begin{array}{c}
\epsilon \\
\left(1+\epsilon \Gamma\left(t, x_{1}\right)\right)
\end{array}\right) \theta, \\
& \sigma\left(t, x_{1}\right)=\left(\begin{array}{cc}
\sigma & 0 \\
\sigma \Gamma\left(t, x_{1}\right) & \delta \sigma \Gamma\left(t, x_{1}\right)
\end{array}\right) .
\end{aligned}
$$

Then, the optimal hedging problem is rewritten as the following minimization problem, where the hedger aims to minimize the expectation of the slippage cost together with the small market impact, the liquidation cost of the underlying asset at maturity, and the rescaled quadratic variation.

$$
\inf _{\theta \in \mathcal{V}} \mathbf{E}\left[\int_{0}^{T} L\left(s, P_{s}, Y_{s}, \theta_{s}\right) d s+\psi\left(P_{T}, Y_{T}\right)\right],
$$

subject to

$$
\left(\begin{array}{l}
d P_{s} \\
d Y_{s}
\end{array}\right)=f\left(s, P_{s}, \theta_{s}\right) d s+\sigma\left(s, P_{s}\right)\left(\begin{array}{c}
d W_{s} \\
d W_{s}^{\perp}
\end{array}\right),
$$

where $\mathcal{V}$ is the set of all admissible controls. Note that this is a generalized form of a linear-quadratic control problem. As a special case, when $\Gamma$ is a constant and $\psi$ is (7) or (8) with a quadratic form of $x_{1}$ and $x_{2}$ (e.g. the variance option in Case 1 where $g_{x_{1}}\left(t, x_{1}\right)=\gamma\left(x_{1}-K\right)$ ), it becomes a linear-quadratic control, and it is reduced to solving a system of ODEs (see Theorem 6.6 in Hanson [8], for instance).

Here, for given $\left(t, x_{1}, x_{2}\right)$, we define an admissible feedback control as a map $\theta:[0, T] \times \mathbf{R}^{2} \rightarrow U, U \subset \mathbf{R}$ satisfying the following.

(1) There exists a two dimensional Brownian Motion $\left(W, W^{\perp}\right)$ such that a solution $\left(Y_{s}, P_{s}\right), t \leq s \leq T$ of the following SDE exists uniquely in law. Namely, a unique weak solution exists for

$$
\begin{gathered}
\left(\begin{array}{l}
d P_{s} \\
d Y_{s}
\end{array}\right)=f\left(s, P_{s}, \theta\left(s, P_{s}, Y_{s}\right)\right) d s+\sigma\left(s, P_{s}\right)\left(\begin{array}{c}
d W_{s} \\
d W_{s}^{\perp}
\end{array}\right), \\
\left(P_{t}, Y_{t}\right)=\left(x_{1}, x_{2}\right) .
\end{gathered}
$$

(2) For any $k>0, \mathbf{E}^{\left(t, x_{1}, x_{2}\right)}\left[\left|\left(P_{s}, Y_{s}\right)\right|^{k}\right]$ is bounded for $t \leq s \leq T$, and $\mathbf{E}^{\left(t, x_{1}, x_{2}\right)}\left[\int_{t}^{T}\left|\theta\left(s, P_{s}, Y_{s}\right)\right|^{k} d s\right]<$
$\infty$.

Next, let us introduce the following HJB equation corresponding to the minimization problem (15): For $u$ : 


$$
\begin{aligned}
& {[0, T] } \times \mathbf{R}^{2} \rightarrow \mathbf{R} \\
& 0= u_{t}+\frac{1}{2} \sigma^{2} u_{x_{1} x_{1}}+\sigma^{2} \Gamma\left(t, x_{1}\right) u_{x_{1} x_{2}} \\
&+\frac{1}{2} \tilde{\sigma}^{2} \Gamma\left(t, x_{1}\right)^{2} u_{x_{2} x_{2}}+\lambda \sigma^{2}\left(x_{2}^{2}+\delta^{2} x_{1}^{2} \Gamma\left(t, x_{1}\right)^{2}\right) \\
&+\inf _{\theta \in U}\left[\left\{\left(1+\epsilon \Gamma\left(t, x_{1}\right)\right) u_{x_{2}}+\epsilon u_{x_{1}}-\epsilon x_{2}\right\} \theta+\eta \theta^{2}\right], \\
& u\left(T, x_{1}, x_{2}\right)=\psi\left(x_{1}, x_{2}\right) .
\end{aligned}
$$

Then, let $\theta^{*}\left(t, x_{1}, x_{2}\right)=-\frac{1}{2 \eta}\left(\left(1+\epsilon \Gamma\left(t, x_{1}\right)\right) u_{x_{2}}\left(t, x_{1}, x_{2}\right)\right.$ $\left.+\epsilon u_{x_{1}}\left(t, x_{1}, x_{2}\right)-\epsilon x_{2}\right)$. When $U=\mathbf{R}$, "inf" is attained at $\theta^{*}$ and then, (17) is rewritten as the semilinear PDE below with explicit dependence on $\epsilon$ :

$$
\begin{aligned}
0= & u_{t}^{(\epsilon)}+\frac{1}{2} \sigma^{2} u_{x_{1}, x_{1}}^{(\epsilon)}+\sigma^{2} \Gamma\left(t, x_{1}\right) u_{x_{1}, x_{2}}^{(\epsilon)} \\
& +\frac{1}{2} \tilde{\sigma}^{2} \Gamma\left(t_{1}, x_{1}\right)^{2} u_{x_{2}, x_{2}}^{(\epsilon)}+\lambda \sigma^{2}\left(x_{2}^{2}+\delta^{2} x_{1}^{2} \Gamma\left(t, x_{1}\right)^{2}\right) \\
& -\frac{1}{4 \eta}\left[\left(1+\epsilon \Gamma\left(t, x_{1}\right)\right) u_{x_{2}}^{(\epsilon)}+\epsilon u_{x_{1}}^{(\epsilon)}-\epsilon x_{2}\right]^{2} \\
& u^{(\epsilon)}\left(T, x_{1}, x_{2}\right)=\psi\left(x_{1}, x_{2}\right) .
\end{aligned}
$$

Particularly, as for variance options with physical and cash settlements, the PDEs become the ones corresponding to linear-quadratic cases. For instance, as seen in Example 1 in Section 5, this is solved explicitly for a variance option in physical settlement.

In other cases such as Example 2 in Section 5, let us assume the existence of a classical solution $u^{(\epsilon)}\left(t, x_{1}, x_{2}\right)$ of (18) with admissibility of $\theta^{*}$. Then, $\theta^{*}$ becomes the solution of the optimal trading problem (15), since a verification theorem holds. (For a verification theorem and sufficient conditions of the existence of a classical solution, see Theorem VI.4.1 and Theorem VI.6.2 in Fleming and Rishel [5], respectively for instance.)

\section{Asymptotic expansion of the semilinear PDE}

In this section, we consider the first order expansion of the classical solution of (18), the Cauchy problem of the second order semilinear PDE of parabolic type, by asymptotic expansion. Let $b_{0}$ and $b_{1}$ be functions of class $C^{2}(\mathbf{R})$ satisfying a polynomial growth and the Lipschitz condition, respectively. We assume that $b_{0}^{\prime}, b_{1}^{\prime}$, and $b_{1}^{\prime \prime}$ satisfy a polynomial growth condition.

In particular, we set the penalty function $\psi$ in (18) as

$$
\psi\left(x_{1}, x_{2}\right)=b_{2} x_{2}^{2}+b_{1}\left(x_{1}\right) x_{2}+b_{0}\left(x_{1}\right), b_{2}>0 .
$$

We consider the following asymptotic expansion of $u^{(\epsilon)}$ with respect to $\epsilon$ around $\epsilon=0$ up to the first order

$$
u^{(0)}+\epsilon u^{(1,0)},
$$

where $u^{(0)}$ and $u^{(1,0)}$ are defined by the following PDEs.

- zeroth order:

$$
\begin{aligned}
0= & u_{t}^{(0)}+\frac{1}{2} \sigma^{2} u_{x_{1}, x_{1}}^{(0)}+\sigma^{2} \Gamma\left(t, x_{1}\right) u_{x_{1}, x_{2}}^{(0)} \\
& +\frac{1}{2} \tilde{\sigma}^{2} \Gamma\left(t_{1}, x_{1}\right)^{2} u_{x_{2}, x_{2}}^{(0)}+\lambda \sigma^{2}\left(x_{2}^{2}+\delta^{2} x_{1}^{2} \Gamma\left(t, x_{1}\right)^{2}\right) \\
& -\frac{1}{4 \eta} u_{x_{2}}^{(0) 2}, u^{(0)}\left(T, x_{1}, x_{2}\right)=b_{2} x_{2}^{2}+b_{1}\left(x_{1}\right) x_{2}+b_{0}\left(x_{1}\right) .
\end{aligned}
$$

- first order:

$$
\begin{aligned}
& 0=u_{t}^{(1,0)}+\frac{1}{2} \sigma^{2} u_{x_{1}, x_{1}}^{(1,0)}+\sigma^{2} \Gamma\left(t, x_{1}\right) u_{x_{1}, x_{2}}^{(1,0)} \\
& +\frac{1}{2} \tilde{\sigma}^{2} \Gamma\left(t, x_{1}\right)^{2} u_{x_{2}, x_{2}}^{(1,0)}-\frac{1}{2 \eta} u_{x_{2}}^{(0)} u_{x_{2}}^{(1,0)}
\end{aligned}
$$

$$
-\frac{1}{2 \eta} u_{x_{2}}^{(0)}\left[\Gamma\left(t, x_{1}\right) u_{x_{2}}^{(0)}+u_{x_{1}}^{(0)}-x_{2}\right], u^{(1,0)}\left(T, x_{1}, x_{2}\right)=0 .
$$

Here, the solution of the semilinear PDE (21) has an explicit form (29) as we will observe in Section 4.1. Existence of a unique solution of the PDE (22) with polynomial growth follows from Remark 5.7.8 in Karatzas and Shreve [10] if $u_{x_{2}}^{(0)}$ is 0 or bounded, which is satisfied in the case of any option in physical settlement or the call option with smooth modification in Case 2 in cash settlement. We will also observe the Feynman-Kac representation of $u^{(1,0)}(37)$ in Section 4.2.

For $\epsilon^{\prime} \in(0, \epsilon]$, the first and second order remainders of the expansion $w^{\left(\epsilon^{\prime}\right)}, v^{\left(\epsilon^{\prime}\right)}$ are defined as follows: $w^{\left(\epsilon^{\prime}\right)} \equiv$ $\frac{1}{\epsilon^{\prime}}\left(u^{\left(\epsilon^{\prime}\right)}-u^{(0)}\right)$, and $v^{\left(\epsilon^{\prime}\right)} \equiv \frac{1}{\epsilon^{\prime 2}}\left(u^{\left(\epsilon^{\prime}\right)}-u^{(0)}-\epsilon^{\prime} u^{(1,0)}\right)$. It follows that $v^{\left(\epsilon^{\prime}\right)}$ satisfies the following PDE,

$$
\begin{aligned}
0= & v_{t}^{\left(\epsilon^{\prime}\right)}+\frac{1}{2} \sigma^{2} v_{x_{1}, x_{1}}^{\left(\epsilon^{\prime}\right)}+\sigma^{2} \Gamma\left(t, x_{1}\right) v_{x_{1}, x_{2}}^{\left(\epsilon^{\prime}\right)}+\frac{1}{2} \tilde{\sigma}^{2} \Gamma\left(t, x_{1}\right)^{2} v_{x_{2}, x_{2}}^{\left(\epsilon^{\prime}\right)} \\
- & \frac{1}{2 \eta}\left\{\left(1+\epsilon^{\prime} \Gamma\left(t, x_{1}\right)\right)\left(u_{x_{2}}^{(0)}+\epsilon^{\prime} u_{x_{2}}^{(1,0)}\right)+\epsilon^{\prime}\left(u_{x_{1}}^{(0)}+\epsilon^{\prime} u_{x_{1}}^{(1,0)}\right)-\epsilon^{\prime} x_{2}\right\} \\
& \cdot\left(\left(1+\epsilon^{\prime} \Gamma\left(t, x_{1}\right)\right) v_{x_{2}}^{\left(\epsilon^{\prime}\right)}+\epsilon^{\prime} v_{x_{1}}^{\left(\epsilon^{\prime}\right)}\right)+h^{\left(\epsilon^{\prime}\right)}\left(t, x_{1}, x_{2}\right), \\
& v^{\left(\epsilon^{\prime}\right)}\left(T, x_{1}, x_{2}\right)=0,
\end{aligned}
$$

where

$$
\begin{aligned}
& h^{\left(\epsilon^{\prime}\right)}\left(t, x_{1}, x_{2}\right) \\
& =-\frac{1}{4 \eta}\left(\Gamma\left(t, x_{1}\right) u_{x_{2}}^{(0)}+u_{x_{2}}^{(1,0)}+u_{x_{1}}^{(0)}-x_{2}\right)^{2} \\
& -\epsilon^{\prime 2} \frac{1}{4 \eta}\left(\Gamma\left(t, x_{1}\right) u_{x_{2}}^{(1,0)}+u_{x_{1}}^{(1,0)}\right)^{2} \\
& -\epsilon^{\prime} \frac{1}{2 \eta}\left(\Gamma\left(t, x_{1}\right) u_{x_{2}}^{(1,0)}+u_{x_{1}}^{(1,0)}\right) \\
& \cdot\left(\Gamma\left(t, x_{1}\right) u_{x_{2}}^{(0)}+u_{x_{2}}^{(1,0)}+u_{x_{1}}^{(0)}-x_{2}\right) \\
& -\frac{1}{2 \eta} u_{x_{2}}^{(0)}\left(\Gamma\left(t, x_{1}\right) u_{x_{2}}^{(1,0)}+u_{x_{1}}^{(1,0)}\right) \\
& -\frac{1}{4 \eta}\left\{\left(w_{x_{2}}^{\left(\epsilon^{\prime}\right)}-u_{x_{2}}^{(1,0)}\right)+\Gamma\left(t, x_{1}\right)\left(u_{x_{2}}^{\left(\epsilon^{\prime}\right)}-u_{x_{2}}^{(0)}-\epsilon^{\prime} u_{x_{2}}^{(1,0)}\right)\right. \\
& \left.+\left(u_{x_{1}}^{\left(\epsilon^{\prime}\right)}-u_{x_{1}}^{(0)}-\epsilon^{\prime} u_{x_{1}}^{(1,0)}\right)\right\}^{2} .
\end{aligned}
$$

In order to obtain an error estimate of (20), we consider the Feynman-Kac representation of $v^{\left(\epsilon^{\prime}\right)}$.

For $\epsilon^{\prime} \in(0, \epsilon]$, let $\alpha^{\left(\epsilon^{\prime}\right)}\left(t, x_{1}, x_{2}\right)=-\frac{1}{2 \eta}\{(1+$ $\left.\epsilon^{\prime} \Gamma\left(t, x_{1}\right)\right)\left(u_{x_{2}}^{(0)}+\epsilon^{\prime} u_{x_{2}}^{(1,0)}\right)$

$$
\left.+\epsilon^{\prime}\left(u_{x_{1}}^{(0)}+\epsilon^{\prime} u_{x_{1}}^{(1,0)}\right)-\epsilon^{\prime} x_{2}\right\} \cdot\left(\begin{array}{c}
\epsilon^{\prime} \\
1+\epsilon^{\prime} \Gamma\left(t, x_{1}\right)
\end{array}\right) .
$$

We assume the following.

Assumption 2 There exists a constant $L_{T}>0$ such that for all $\epsilon^{\prime} \in(0, \epsilon], t \in[0, T]$, and $\mathbf{x} \in \mathbf{R}^{2}$,

$$
\begin{aligned}
& \left\|\alpha^{\left(\epsilon^{\prime}\right)}(t, \mathbf{x})-\alpha^{\left(\epsilon^{\prime}\right)}\left(t, \mathbf{x}^{\prime}\right)\right\| \leq L_{T}\left\|\mathbf{x}-\mathbf{x}^{\prime}\right\|, \\
& \left\|\alpha^{\left(\epsilon^{\prime}\right)}(t, \mathbf{x})\right\|^{2} \leq L_{T}\left(\|\mathbf{x}\|^{2}+1\right)
\end{aligned}
$$

where $\mathbf{x}=\left(x_{1}, x_{2}\right)$ and $\mathbf{x}^{\prime}=\left(x_{1}^{\prime}, x_{2}^{\prime}\right)$.

Assumption 3 The following term satisfies a polynomial growth condition uniformly on $\epsilon^{\prime} \in(0, \epsilon]$. That is, there exists $D>0$ and $k \in \mathbf{N}$ such that for all $\epsilon^{\prime} \in(0, \epsilon]$, $t \in[0, T]$, and $\mathbf{x} \in \mathbf{R}^{2}$,

$$
\left|h^{\left(\epsilon^{\prime}\right)}(t, \mathbf{x})\right| \leq D\left(1+|\mathbf{x}|^{k}\right) \text {. }
$$

Note that $\sigma\left(t, x_{1}\right)$ satisfies the Lipschitz and linear growth condition and Assumption 1. From Assumptions 2 and 3, and Theorem 5.7.6 in Karatzas and Shreve [10], 
$v^{\left(\epsilon^{\prime}\right)}$ has a Feynman-Kac representation,

$$
v^{\left(\epsilon^{\prime}\right)}(t, \mathbf{x})=\mathbf{E}^{(t, \mathbf{x})}\left[\int_{t}^{T} h^{\left(\epsilon^{\prime}\right)}\left(s, \mathbf{X}_{s}\right) d s\right]
$$

where $\mathbf{X}_{t}=\left(X_{1, t}, X_{2, t}\right)$,

$$
\left(\begin{array}{l}
d X_{1, t} \\
d X_{2, t}
\end{array}\right)=\alpha^{\left(\epsilon^{\prime}\right)}\left(t, X_{1, t}, X_{2, t}\right) d t+\sigma\left(t, X_{1, t}\right)\left(\begin{array}{l}
d W_{1, t} \\
d W_{2, t}
\end{array}\right),
$$

and the following estimation holds.

Theorem 1 Under Assumption 2 and 3, there exists $C>0$ and $k \in \mathbf{N}$ such that $\left|v^{\left(\epsilon^{\prime}\right)}(t, \mathbf{x})\right|<C\left(1+|\mathbf{x}|^{2 k}\right)$, for all $\epsilon^{\prime} \in(0, \epsilon], t \in[0, T]$, and $\mathbf{x} \in \mathbf{R}^{2}$

Proof. $\left|v^{\left(\epsilon^{\prime}\right)}(t, \mathbf{x})\right|=\left|\mathbf{E}^{(t, \mathbf{x})}\left[\int_{t}^{T} h^{\left(\epsilon^{\prime}\right)}\left(s, \mathbf{X}_{s}\right) d s\right]\right| \leq$ $\mathbf{E}^{(t, \mathbf{x})}\left[\int_{t}^{T} D\left(1+\left|\mathbf{X}_{s}\right|^{2 k}\right) d s\right] \leq D C_{2 k, T}\left(1+|\mathbf{x}|^{2 k}\right)(T-t)$. In the last inequality, we have used the moment estimation result on a solution of SDEs. (e.g. Theorem V.4.2 in Fleming and Rishel [5].)

Remark 4 Theorem 1 shows that for $\epsilon^{\prime} \in(0, \epsilon]$, the remainder of the first order expansion $(n=1)$ is $\mathcal{O}\left(\epsilon^{\prime 2}\right)$, that is,

$$
\limsup _{\epsilon^{\prime} \downarrow 0} \frac{\left|u^{\left(\epsilon^{\prime}\right)}-u^{(0)}-u^{(1,0)}\right|}{\epsilon^{\prime 2}}<\infty .
$$

\section{Computation of coefficients in the expansion}

This section calculates the zeroth and the first order coefficient of the expansion in (21) and (22).

\section{$4.1 u^{(0)}\left(t, x_{1}, x_{2}\right)$}

We assume that $u^{(0)}$, a solution of the semilinear PDE (21), has the following expression, that is, in fact the unique solution:

$$
\begin{aligned}
& u^{(0)}\left(t, x_{1}, x_{2}\right)=B_{2}(t) x_{2}^{2}+B_{1}\left(t, x_{1}\right) x_{2}+B_{0}\left(t, x_{1}\right), \\
& u^{(0)}\left(T, x_{1}, x_{2}\right)=b_{2} x_{2}^{2}+b_{1}\left(x_{1}\right) x_{2}+b_{0}\left(x_{1}\right), b_{2}>0,
\end{aligned}
$$

where $B_{2}, B_{1}$ and $B_{0}$ are unique solutions of the following system of ODE and PDEs

$$
\begin{gathered}
0=B_{2}^{\prime}(t)-\frac{1}{\eta} B_{2}^{2}(t)+\lambda \sigma^{2}, B_{2}(T)=b_{2}, \\
-B_{1, t}\left(t, x_{1}\right)+\frac{1}{\eta} B_{2}(t) B_{1}\left(t, x_{1}\right)=+\frac{1}{2} \sigma^{2} B_{1, x_{1} x_{1}}\left(t, x_{1}\right), \\
B_{1}\left(T, x_{1}\right)=b_{1}\left(x_{1}\right), \\
-B_{0, t}\left(t, x_{1}\right)=+\frac{1}{2} \sigma^{2} B_{0, x_{1} x_{1}}\left(t, x_{1}\right)+f\left(t, x_{1}\right), \\
B_{0}\left(T, x_{1}\right)=b_{0}\left(x_{1}\right),
\end{gathered}
$$

where $f\left(t, x_{1}\right)=\sigma^{2} \Gamma\left(t, x_{1}\right) B_{1, x_{1}}\left(t, x_{1}\right)+\tilde{\sigma}^{2} \Gamma\left(t, x_{1}\right)^{2} B_{2}(t)-$ $\frac{1}{4 \eta} B_{1}\left(t, x_{1}\right)^{2}+\delta^{2} \lambda \sigma^{2} x_{1}^{2} \Gamma\left(t, x_{1}\right)^{2}$. Note that the ODE

(30) is a Riccati type and has the unique solution

$$
B_{2}(t)=
$$$$
\left\{\begin{array}{l}
\sqrt{\lambda \eta \sigma^{2}} \tanh \left(-\frac{1}{2} \log \frac{1-h_{0}}{1+h_{0}}+\sqrt{\frac{\lambda \sigma^{2}}{\eta}}(T-t)\right), \\
\text { if } 0<b_{2}<\sqrt{\lambda \eta \sigma^{2}}, \\
\sqrt{\lambda \eta \sigma^{2}}, \text { if } b_{2}=\sqrt{\lambda \eta \sigma^{2}}, \\
\sqrt{\lambda \eta \sigma^{2}} \operatorname{coth}\left(-\frac{1}{2} \log \frac{h_{0}-1}{h_{0}+1}+\sqrt{\frac{\lambda \sigma^{2}}{\eta}}(T-t)\right), \\
\text { if } \sqrt{\lambda \eta \sigma^{2}}<b_{2},
\end{array}\right.
$$

where $h_{0}=\frac{b_{2}}{\sqrt{\lambda \eta \sigma^{2}}}$.

Note that the PDEs (31) and (32) have a unique solution with Feynman-Kac representation as follows.

Since $B_{2}(t)$ is bounded from above and below and $b_{1}$ satisfies a polynomial growth condition, by Remark 5.7.8 in Karatzas and Shreve [10], the PDE (31) has a solution of class $C^{1,2}$ with polynomial growth. Then by Theorem 4.4.2 in Karatzas and Shreve [10], $B_{1}$ has a FeynmanKac representation.

For the PDE (32), the nonhomogeneous term $f\left(t, x_{1}\right)$ is locally Hölder continuous uniformly in $t$, which follows from the fact that the first order derivative with respect to $x_{1}$ is bounded uniformly in $t$ on any compact subset of R. This point as well as the polynomial growth condition on $f\left(t, x_{1}\right)$ can be checked by (34) below, Assumption 1 and the linear/polynomial growth condition on $b_{1}, b_{1}^{\prime}$ and $b_{1}^{\prime \prime}$. Then by Remark 5.7.8 in Karatzas and Shreve [10], the PDE (32) has a solution of class $C^{1,2}$ with polynomial growth and $B_{0}$ also has a Feynman-Kac representation.

First, for $B_{1}, B_{1, x_{1}}$ and $B_{1, x_{1} x_{1}}$,

$$
\begin{gathered}
B_{1}\left(t, x_{1}\right)=e^{-\int_{t}^{T} \frac{1}{\eta} B_{2}(s) d s} \mathbf{E}\left[b_{1}\left(x_{1}+\sigma W_{T-t}\right)\right], \\
B_{1, x_{1}}\left(t, x_{1}\right)=e^{-\int_{t}^{T} \frac{1}{\eta} B_{2}(s) d s} \mathbf{E}\left[b_{1}^{\prime}\left(x_{1}+\sigma W_{T-t}\right)\right], \\
B_{1, x_{1} x_{1}}\left(t, x_{1}\right)=e^{-\int_{t}^{T} \frac{1}{\eta} B_{2}(s) d s} \mathbf{E}\left[b_{1}^{\prime \prime}\left(x_{1}+\sigma W_{T-t}\right)\right] .
\end{gathered}
$$

By (33), it follows that

$$
e^{-\int_{t}^{T} \frac{1}{\eta} B_{2}(s) d s}
$$

$$
=\left\{\begin{array}{l}
\frac{\cosh \left(-\frac{1}{2} \log \frac{1-h_{0}}{1+h_{0}}\right)}{\cosh \left(-\frac{1}{2} \log \frac{1-h_{0}}{1+h_{0}}+\sqrt{\frac{\lambda \sigma^{2}}{\eta}}(T-t)\right)}, \text { if } 0<b_{2}<\sqrt{\lambda \eta \sigma^{2}}, \\
\exp \left(-\sqrt{\left.\frac{\lambda \sigma^{2}}{\eta}(T-t)\right), \text { if } b_{2}=\sqrt{\lambda \eta \sigma^{2}}}\right. \\
\frac{\sinh \left(-\frac{1}{2} \log \frac{h_{0}-1}{h_{0}+1}\right)}{\sinh \left(-\frac{1}{2} \log \frac{h_{0}-1}{h_{0}+1}+\sqrt{\frac{\lambda \sigma^{2}}{\eta}}(T-t)\right)}, \text { if } \sqrt{\lambda \eta \sigma^{2}}<b_{2} .
\end{array}\right.
$$

Next, as for $B_{0}$,

$$
\begin{aligned}
& B_{0}\left(t, x_{1}\right)=\mathbf{E}\left[b_{0}\left(x_{1}+\sigma W_{T-t}\right)\right. \\
& +\int_{t}^{T}\left(\sigma^{2} \Gamma\left(s, x_{1}+\sigma W_{s-t}\right) B_{1, x_{1}}\left(s, x_{1}+\sigma W_{s-t}\right)\right. \\
& +\tilde{\sigma}^{2} \Gamma\left(s, x_{1}+\sigma W_{s-t}\right)^{2} B_{2}(s)-\frac{1}{4 \eta} B_{1}\left(s, x_{1}+\sigma W_{s-t}\right)^{2} \\
& \left.\left.\quad+\delta^{2} \lambda \sigma^{2}\left(x_{1}+\sigma W_{s-t}\right)^{2} \Gamma\left(s, x_{1}+\sigma W_{s-t}\right)^{2}\right) d s\right]
\end{aligned}
$$

Then $B_{0, x_{1}}$ is given by

$$
\begin{aligned}
& B_{0, x_{1}}\left(t, x_{1}\right)=\mathbf{E}\left[b_{0}^{\prime}\left(x_{1}+\sigma W_{T-t}\right)\right. \\
& \quad+\int_{t}^{T}\left(\sigma ^ { 2 } \left\{\Gamma_{x_{1}}\left(s, x_{1}+\sigma W_{s-t}\right) B_{1, x_{1}}\left(s, x_{1}+\sigma W_{s-t}\right)\right.\right. \\
& \left.\quad+\Gamma\left(s, x_{1}+\sigma W_{s-t}\right) B_{1, x_{1} x_{1}}\left(s, x_{1}+\sigma W_{s-t}\right)\right\} \\
& \quad+2 \tilde{\sigma}^{2} \Gamma\left(s, x_{1}+\sigma W_{s-t}\right) \Gamma_{x_{1}}\left(s, x_{1}+\sigma W_{s-t}\right) B_{2}(s) \\
& \quad-\frac{1}{2 \eta} B_{1}\left(s, x_{1}+\sigma W_{s-t}\right) B_{1, x_{1}}\left(s, x_{1}+\sigma W_{s-t}\right) \\
& +2 \delta^{2} \lambda \sigma^{2}\left(x_{1}+\sigma W_{s-t}\right) \Gamma^{2}\left(s, x_{1}+\sigma W_{s-t}\right) \\
& +2 \delta^{2} \lambda \sigma^{2}\left(x_{1}+\sigma W_{s-t}\right)^{2} \\
& \left.\left.+\Gamma\left(s, x_{1}+\sigma W_{s-t}\right) \Gamma_{x_{1}}\left(s, x_{1}+\sigma W_{s-t}\right)\right) d s\right] .
\end{aligned}
$$




\section{2 $u^{(1,0)}\left(t, x_{1}, x_{2}\right)$}

Finally, since the Lipschitz \& linear growth condition hold for $u_{x_{2}}^{(0)}$ because of (29),(34) and the Lipschitz condition on $b_{1}$, by Theorem 5.7.6 in Karatzas and Shreve [10], $u^{(1,0)}$ has the following Feynman-Kac representation.

$$
\begin{aligned}
& u^{(1,0)}\left(t, x_{1}, x_{2}\right)=\mathbf{E}^{\left(t, x_{1}, x_{2}\right)}\left[\int_{t}^{T}-\frac{1}{2 \eta} u_{x_{2}}^{(0)}\left(s, X_{1, s}, X_{2, s}\right)\right. \\
& \cdot\left\{\Gamma\left(s, X_{1, s}\right) u_{x_{2}}^{(0)}\left(s, X_{1, s}, X_{2, s}\right)+u_{x_{1}}^{(0)}\left(s, X_{1, s}, X_{2, s}\right)\right. \\
& \left.\left.\quad-X_{2, s}\right\} d s\right],
\end{aligned}
$$

where $d X_{1, s}=\sigma d W_{1, s}, \quad d X_{2, s}=\sigma \Gamma\left(s, X_{1, s}\right)\left(d W_{1, s}+\right.$ $\left.\delta d W_{2, s}\right)-\frac{1}{2 \eta} u_{x_{2}}^{(0)}\left(s, X_{1, s}, X_{2, s}\right) d s$.

Note that by $(29), u^{(0)}\left(t, x_{1}, x_{2}\right)=B_{2}(t) x_{2}^{2}+B_{1}\left(t, x_{1}\right) x_{2}+$ $B_{0}\left(t, x_{1}\right), u_{x_{2}}^{(0)}\left(t, x_{1}, x_{2}\right)=2 B_{2}(t) x_{2}+B_{1}\left(t, x_{1}\right)$, and $u_{x_{1}}^{(0)}\left(t, x_{1}, x_{2}\right)=B_{1, x_{1}}\left(t, x_{1}\right) x_{2}+B_{0, x_{1}}\left(t, x_{1}\right)$, where $B_{2}, B_{1}, B_{1, x_{1}}, B_{0}$ and $B_{0, x_{1}}$ are calculated in Section 4.1 .

\subsection{Derivatives pricing and optimal hedging strategy}

Taking the objective function for maximization (14) into consideration, we define a derivatives price as the largest $m$ satisfying the following inequality for some threshold $\tilde{K} \geq 0, g\left(0, P_{0}\right)-m-u\left(0, P_{0}, Y_{0}\right) \geq \tilde{K}$, that is, $g\left(0, P_{0}\right)-$ $u\left(0, P_{0}, Y_{0}\right)-\tilde{K}$. In other words, the derivatives price for the buyer is the highest premium which the trader is willing to pay in order to make profit $\tilde{K}$. Conversely, the derivatives price for the seller is the lowest premium which the trader is willing to receive to gain $\tilde{K}$. Note that by $(6), Y_{0}=0$ (i.e. $\tilde{X}_{0}=-g_{x_{1}}\left(t, P_{0}\right)$ ) when there is an initial delta exchange with the counter party, and $Y_{0}=g_{x_{1}}\left(t, P_{0}\right)$ (i.e. $\left.\tilde{X}_{0}=0\right)$ when there is no initial exchange.

We also note that the hedging cost we mentioned in Section 2.4 corresponds to $u\left(0, P_{0}, Y_{0}\right)$ when $\tilde{K}=0$. Hereafter, we consider the derivatives price and the hedging cost when $\tilde{K}=0$.

\section{$5 \quad$ Examples}

This section presents examples of the hedging cost $u^{(\epsilon)}$ in (18) with $\delta=0$ for the variance option in Case 1 , and the asymptotic expansion in (20) for the European call option corresponding to Case 2 in physical settlement with allowing $\delta \neq 0$. The asymptotic expansion for the European call option in cash settlement and the variance option in physical settlement are given in Appendices A and B, respectively.

Example 1. As for the payoff $h\left(x_{1}\right)=\frac{1}{2} \gamma x_{1}^{2}+c_{1} x_{1}+$ $c_{0}$ in Case 1 for the physical settlement where $b_{2}>0$, $b_{1}=b_{0}=0$ with $\delta=0, u\left(t, x_{1}, x_{2}\right)$ in (18) is solved explicitly as follows. We assume that $\epsilon \gamma \neq-1$. Let $h_{0}=$ $\frac{(2+2 \gamma \epsilon) b_{2}-\epsilon}{2 \sqrt{\lambda \eta \sigma^{2}}}$.

$$
u^{(\epsilon)}\left(t, x_{1}, x_{2}\right)=A_{2}^{(\epsilon)}(T-t) x_{2}^{2}+A_{0}^{(\epsilon)}(T-t),
$$

$$
\begin{aligned}
& A_{2}^{(\epsilon)}\left(t^{\prime}\right)= \\
& \int \frac{1}{2+2 \gamma \epsilon}\left\{2 \sqrt{\lambda \eta \sigma^{2}} \tanh \left(-\frac{1}{2} \log \frac{1-h_{0}}{1+h_{0}}+\sqrt{\frac{(2+2 \gamma \epsilon)^{2} \lambda \sigma^{2}}{4 \eta}} t^{\prime}\right)\right. \\
& +\epsilon\} \text {, if }-1<h_{0}<1 \\
& \left\{\frac{1}{2+2 \gamma \epsilon}\left(2 \sqrt{\lambda \eta \sigma^{2}}+\epsilon\right) \text {, if } h_{0}=1\right. \text {, } \\
& \frac{1}{2+2 \gamma \epsilon}\left\{2 \sqrt{\lambda \eta \sigma^{2}} \operatorname{coth}\left(-\frac{1}{2} \log \frac{h_{0}-1}{h_{0}+1}+\sqrt{\frac{(2+2 \gamma \epsilon)^{2} \lambda \sigma^{2}}{4 \eta}} t^{\prime}\right)\right. \\
& +\epsilon\} \text {, if } h_{0}>1 \\
& A_{0}^{(\epsilon)}\left(t^{\prime}\right)= \\
& \left\{\begin{array}{l}
\frac{4 \gamma^{2} \sigma^{2} \eta}{(2+2 \gamma \epsilon)^{2}} \log \frac{\cosh \left(-\frac{1}{2} \log \frac{1-h_{0}}{1+h_{0}}+\sqrt{\frac{(2+2 \gamma \epsilon)^{2} \lambda \sigma^{2}}{4 \eta}} t^{\prime}\right)}{\cosh \left(-\frac{1}{2} \log \frac{1-h_{0}}{1+h_{0}}\right)}+\frac{\gamma^{2} \sigma^{2} \epsilon}{(2+2 \gamma \epsilon)} t^{\prime}, \\
\text { if }-1<h_{0}<1, \\
\frac{\gamma^{2} \sigma^{2}}{2+2 \gamma \epsilon}\left(2 \sqrt{\lambda \eta \sigma^{2}}+\epsilon\right) t^{\prime}, \text { if } h_{0}=1, \\
\frac{4 \gamma^{2} \sigma^{2} \eta}{(2+2 \gamma \epsilon)^{2}} \log \frac{\sinh \left(-\frac{1}{2} \log \frac{h_{0}-1}{h_{0}+1}+\sqrt{\frac{(2+2 \gamma \epsilon)^{2} \lambda \sigma^{2}}{4 \eta}} t^{\prime}\right)}{\sinh \left(-\frac{1}{2} \log \frac{h_{0}-1}{h_{0}+1}\right)}+\frac{\gamma^{2} \sigma^{2} \epsilon}{(2+2 \gamma \epsilon)} t^{\prime}, \\
\text { if } h_{0}>1 .
\end{array}\right.
\end{aligned}
$$

Example 2. In physical settlement of a call option, where $b_{2}>0, b_{1}=b_{0}=0$ and $h\left(x_{1}\right)=\left(x_{1}-K\right)^{+}$, for fixed $0 \leq t \leq T$, we have $B_{1}=B_{1, x_{1}}=B_{1, x_{1} x_{1}}=0$, $g\left(0, x_{1}\right)=\frac{\sigma \sqrt{T-t}}{\sqrt{2 \pi}} e^{-\frac{\left(x_{1}-K\right)^{2}}{2 \sigma^{2}(T-t)}}+\left(x_{1}-K\right) N\left(\frac{x_{1}-K}{\sigma \sqrt{T-t}}\right)$, $\Gamma\left(s, x_{1}\right)=\frac{1}{\sqrt{2 \pi \sigma^{2}(T-s)}} e^{-\frac{\left(x_{1}-K\right)^{2}}{2 \sigma^{2}(T-s)}}, t \leq \forall s<T$.

It follows that $\mathbf{E}\left[\Gamma^{2}\left(s, x_{1}+\sigma W_{s-t}\right)\right]=\frac{1}{2 \pi \sigma^{2} \sqrt{T-s} \sqrt{T+s-2 t}}$ $\exp \left(-\frac{\left(x_{1}-K\right)^{2}}{\sigma^{2}(T+s-2 t)}\right), \mathbf{E}\left[\left(x_{1}+\sigma W_{s-t}\right)^{2} \Gamma^{2}\left(s, x_{1}+\sigma W_{s-t}\right)\right]$ $=\frac{1}{2 \pi \sigma^{2} \sqrt{T-s} \sqrt{T+s-2 t}} \exp \left(-\frac{\left(x_{1}-K\right)^{2}}{\sigma^{2}(T+s-2 t)}\right)\left\{\frac{\sigma^{2}(s-t)(T-s)}{T+s-2 t}\right.$ $\left.+\left(x_{1}-\frac{2\left(x_{1}-K\right)(s-t)}{T+s-2 t}\right)^{2}\right\}$. By (35), we have $B_{0}\left(t, x_{1}\right)$

$$
\begin{aligned}
\int_{t}^{T} \frac{1}{2 \pi \sigma^{2} \sqrt{T-s} \sqrt{T+s-2 t}} \exp \left(-\frac{\left(x_{1}-K\right)^{2}}{\sigma^{2}(T+s-2 t)}\right) \\
\times\left[\tilde{\sigma}^{2} B_{2}(s)+\delta^{2} \lambda \sigma^{2}\left\{\frac{\sigma^{2}(s-t)(T-s)}{T+s-2 t}\right.\right. \\
\left.\left.+\left(x_{1}-\frac{2\left(x_{1}-K\right)(s-t)}{T+s-2 t}\right)^{2}\right\}\right] d s
\end{aligned}
$$

where $B_{2}(t)$ is given as (33) in Section 4.1.

$$
\begin{aligned}
& B_{0, x_{1}}\left(t, x_{1}\right) \\
& =\int_{t}^{T}-\frac{\left(x_{1}-K\right)}{\pi \sigma^{4} \sqrt{T-s}(\sqrt{T+s-2 t})^{3}} \exp \left(-\frac{\left(x_{1}-K\right)^{2}}{\sigma^{2}(T+s-2 t)}\right) \\
& \quad \times\left[\tilde{\sigma}^{2} B_{2}(s)+\delta^{2} \lambda \sigma^{2}\left\{\frac{\sigma^{2}(s-t)(T-s)}{T+s-2 t}\right.\right. \\
& \left.\left.\quad+\left(x_{1}-\frac{2\left(x_{1}-K\right)(s-t)}{T+s-2 t}\right)^{2}\right\}\right] d s \\
& +\int_{t}^{T} \frac{\delta^{2} \lambda \sqrt{T-s}}{\pi(\sqrt{T+s-2 t})^{3}}\left(x_{1}-\frac{2\left(x_{1}-K\right)(s-t)}{T+s-2 t}\right) \\
& \quad \cdot \exp \left(-\frac{\left(x_{1}-K\right)^{2}}{\sigma^{2}(T+s-2 t)}\right) d s .
\end{aligned}
$$


Note that $u^{(0)}\left(t, x_{1}, x_{2}\right)=B_{2}(t) x_{2}^{2}+B_{0}\left(t, x_{1}\right)$, $u_{x_{2}}^{(0)}\left(t, x_{1}, x_{2}\right)=2 B_{2}(t) x_{2}, u_{x_{1}}^{(0)}\left(t, x_{1}, x_{2}\right)=B_{0, x_{1}}\left(t, x_{1}\right)$. Then, we have

$$
\begin{aligned}
u^{(1,0)}\left(t, x_{1}, x_{2}\right) & \mathbf{E}^{\left(t, x_{1}, x_{2}\right)}\left[\int_{t}^{T}-\frac{1}{\eta} B_{2}(s) X_{2, s}\right. \\
& \left.\cdot\left\{\Gamma\left(s, X_{1, s}\right) 2 B_{2}(s) X_{2, s}+B_{0, x_{1}}\left(s, X_{1, s}\right)-X_{2, s}\right\} d s\right],
\end{aligned}
$$

where $X_{1, s}=x_{1}+\sigma \int_{t}^{s} d W_{1, v}$,

$X_{2, s}=x_{2}+\int_{t}^{s} \sigma \Gamma\left(v, X_{1, v}\right)\left(d W_{1, v}+\delta d W_{2, v}\right)-$ $\int_{t}^{s} \frac{1}{\eta} B_{2}(v) X_{2, v} d v$.

\section{Numerical experiments}

In this section, we show numerical examples of the derivatives prices in Section 5, namely, the prices for the variance option and the European call option in physical settlement.

Firstly, after Section 6.1 explains the set up of the parameters in the numerical examples, Section 6.2 presents the first and the second order error of the asymptotic expansion for the variance option in physical settlement by comparing with the exact solution derived in Example 1 of Section 5. Then, Section 6.3 estimates the first order error for the European call option from that for the variance option which replicates the European call option. We take this approach since it is hard to numerically solve the semilinear PDE (18) directly and properly. ${ }^{1}$

Finally, Section 6.4 presents comparative statics in terms of $\eta, \lambda, T, \sigma$, and $\delta$ for the variance option and the European call option in physical settlement. Hereafter, we denote $u^{(0)}$ by $u_{0}$ and $u^{(1,0)}$ by $u_{1}$.

\subsection{Parameters in the numerical experiments}

In the numerical examples, we use the following parameters as default. $P_{0}=1.00, \sigma=0.20, \delta=0, t=0$, $T=0.50, x_{1}=1.00, K=1.00, x_{2}=0, \epsilon=0.10$, $\lambda=10, \eta=1.60 * 10^{-5}$, and $b_{2}=0.05$. We also set one year as the unit of time. Then, we consider the following two types of derivatives payoffs: Variance option $\left(P_{T}-K\right)^{2}$ and European call option $\left(P_{T}-K\right)^{+}$. For example, suppose that one unit of the notional for the underlying asset is 100 million and all the prices are expressed in USD. Then, the payouts are USD 4 million for the variance option and USD 20 million for the European call option when $P_{T}=1.20$. Also, they are USD 4 million for the variance option and nil for the European call option when $P_{T}=0.80$. We determine the levels of the default parameters as follows.

- Volatility: We set $\sigma=0.20$ so that the instantaneous standard deviation of the price process at $t=0$ corresponds to $20 \%$ of the volatility of the log-normal model (since $P_{0}=1.00$ in our case).

- Market impact parameter: First note that the permanent market impact is $\epsilon \int_{0}^{t} \theta_{s} d s$ in $P_{t}$ as in (1). Suppose that the hedger trades one unit of the underlying asset

\footnotetext{
1 For instance, to the best of our knowledge, Monte Carlo methods for solving a corresponding quadratic backward stochastic differential equations (BSDEs) with unbounded terminals has not yet been sufficiently developed for our purpose.
}

by $t$ and its permanent market impact is 0.10 , which is $10 \%$ of $P_{0}$. Then $\epsilon=0.10$, since $0.10=\epsilon * 1.00$.

- Slippage on the price: The slippage on the price at $t$ is $\eta \theta_{t}$ as in (2), where $\theta_{t}$ is the speed of order placement, the number of units of orders the hedger submits in a unit of time. For example, suppose that the hedger submits 0.1 units of buying orders for the underlying asset in one hour, which is $1.6 * 10^{-4}$ years and the price slippage is 0.01 . Then $0.01=\eta * 0.10 /\left(1.60 * 10^{-4}\right)$, and $\eta=1.60 * 10^{-5}$.

- Liquidation cost at maturity: When the hedger liquidates $\alpha$ units of the underlying asset at maturity immediately, the price spread for the liquidation is $b_{2} \alpha$ as in (8). Suppose that the third party charges 0.05 of the price spread when undertaking one unit of the underlying asset from the hedger. In this case, since $b_{2}$ is the liquidation cost per unit of notional, $b_{2}=0.05$.

- Slippage on the execution volume: The execution slippage at time $t$ is $\delta \sigma \Gamma\left(t, P_{t}\right) d W_{t}^{\perp}$ as in (4). $\delta \sigma \Gamma\left(t, P_{t}\right)$, the coefficient of $d W_{t}^{\perp}$, is $\delta$ times of $\sigma \Gamma\left(t, P_{t}\right)$, which corresponds to the coefficient of $d W_{t}$ for the delta rebalancing amount $\sigma \Gamma\left(t, P_{t}\right) d W_{t}$ in the Bachelier model. When considering the slippage in the numerical example, we set $\delta=0.01$, for example.

\subsection{Asymptotic expansion error for variance option}

In relation to the error estimate of the first order asymptotic expansion in Section 3, we present the first order error of the asymptotic expansion numerically in the case of the variance option. Table 1 shows the values of $u^{(\epsilon)}$ and $u_{0}+\epsilon u_{1}$ for the variance option when $\epsilon=$ $0.01,0.05,0.10$, and 0.20 . We observe that the first order errors are $1 \%, 9 \%$, and $19 \%$ of $u^{(\epsilon)}$ for $\epsilon=0.01,0.05$, and 0.10 , when the proportions of $u^{(\epsilon)}$ to the Bachelier price are $3 \%, 10 \%$ and $18 \%$, respectively. In other words, the first order expansion accounts for $99 \%, 91 \%$ and $81 \%$ of the exact hedging cost when it is $3 \%, 10 \%$, and $18 \%$ of the Bachelier price. We also note that the first order error and the proportion of the hedging cost to the Bachelier price become higher as $\epsilon$ increases. This asymptotic method provides sufficient accuracy for practical use even when $\epsilon=0.10$, which is 7.5 times as high as the one used in the example in Guéant and $\mathrm{Pu}$ [7] (i.e. $7.5=0.10 / 0.01333$, see Section 7.1). Note that we set $b_{2}=0.08$ only for $\epsilon=0.20$ so that $h_{0}>-1$. This condition is necessary for $A_{2}^{(\epsilon)}$ and $A_{0}^{(\epsilon)}$ in (38) in Example 1 to be calculated.

Moreover, we have calculated the second order expansion for the variance option based on the method in Appendix D. We observe that the second order expansion presents better approximation results than the first order expansion as expected.

\begin{tabular}{|l|l|l|lllll|ccc|}
\hline$\epsilon$ & Bachelier & $u^{(\epsilon)}$ & $u_{0}$ & $u_{1}$ & $u_{2}$ & 1st approx & 2nd approx & Error & Error & $u^{(\epsilon)} /$ Bachelier \\
\hline 0.01 & $2.00 \mathrm{E}-02$ & $5.96 \mathrm{E}-04$ & $2.08 \mathrm{E}-04$ & $3.96 \mathrm{E}-02$ & $-1.58 \mathrm{E}-01$ & $6.04 \mathrm{E}-04$ & $5.96 \mathrm{E}-04$ & $1 \%$ & $0 \%$ & $3 \%$ \\
0.05 & $2.00 \mathrm{E}-02$ & $2.01 \mathrm{E}-03$ & $2.08 \mathrm{E}-04$ & $3.96 \mathrm{E}-02$ & $-1.58 \mathrm{E}-01$ & $2.19 \mathrm{E}-03$ & $1.99 \mathrm{E}-03$ & $9 \%$ & $-1 \%$ & $10 \%$ \\
0.1 & $2.00 \mathrm{E}-02$ & $3.50 \mathrm{E}-03$ & $2.08 \mathrm{E}-04$ & $3.96 \mathrm{E}-02$ & $-1.58 \mathrm{E}-01$ & $4.16 \mathrm{E}-03$ & $3.38 \mathrm{E}-03$ & $19 \%$ & $-4 \%$ & $18 \%$ \\
0.2 & $2.00 \mathrm{E}-02$ & $5.86 \mathrm{E}-03$ & $2.10 \mathrm{E}-04$ & $3.96 \mathrm{E}-02$ & $-1.58 \mathrm{E}-01$ & $8.12 \mathrm{E}-03$ & $4.96 \mathrm{E}-03$ & $39 \%$ & $-15 \%$ & $29 \%$ \\
\hline
\end{tabular}

Table 1

Exact and approximation values of $u^{(\epsilon)}$ for variance option, 1st approx $=u_{0}+\epsilon u_{1}, 2$ nd approx $=u_{0}+\epsilon u_{1}+\frac{1}{2} \epsilon^{2} u_{2}$

\subsection{Asymptotic Expansion error for Call option}

Next, we estimate the first order error of the asymptotic expansion of $u^{(\epsilon)}$ for the European call option by approximating the payoff by a variance option $h\left(x_{1}\right)=$ 
$\frac{1}{2} \gamma x_{1}^{2}+c_{1} x_{1}+c_{0}$ in Example 1 in Section 5. We first choose $\gamma$ in the variance option in Example 1 so that $u_{1}$ matches. Since $u_{1}$ and $u_{0}$ only include $\gamma$ (do not either $c_{1}$ or $\left.c_{0}\right), u_{0}$ of the variance option does not coincide with that of the European call option. We select $c_{1}$ and $c_{0}$ so that the Bachelier price $g\left(0, P_{0}\right)$ (i.e. $g\left(t, x_{1}\right)$ in Section 2.2 with $t=0$ and $\left.x_{1}=P_{0}\right)$ and the Bachelier delta $g_{x_{1}}\left(0, P_{0}\right)$ of the variance option agree with those of the European call option.

Tables 2, 3 and 4 show $u_{0}+\epsilon u_{1}$ for the European call option, and $u^{(\epsilon)}$ and $u_{0}+\epsilon u_{1}$ for its replicating variance option for $\epsilon=0.01,0.05$, and 0.10 , respectively.

We estimate the first order errors of the European call option as 2.85E-05, 6.36E-04, and 2.24E-03 for $\epsilon=0.01,0.05$ and 0.10 , which are the corresponding first order error $u^{(\epsilon)}-\left(u_{0}+\epsilon u_{1}\right)$ of the replicating variance option. Hence, the exact values of the hedging cost for the European call option are also estimated as 1.89E-03, 5.07E-03, and 8.20E-03 for $\epsilon=0.01,0.05$ and 0.10 , which are the values of $u_{0}+\epsilon u_{1}$ for the European call option plus the estimated errors $u^{(\epsilon)}-\left(u_{0}+\epsilon u_{1}\right)$ from the replicating variance option. Note that $\gamma=3.105, c_{1}=-2.605$, and $c_{0}=1.078$ in these examples, and due to the large coefficient $\gamma$ of the variance option, the first order error in Table 4 is larger than that in Table 1 when $\epsilon=0.10$.

\begin{tabular}{|l|l|l|lll|lc|}
\hline & Bachelier & $u^{(\epsilon)}$ & $u_{0}$ & $u_{1}$ & $u_{0}+\epsilon u_{1}$ & Error & $u^{(\epsilon)} /$ Bachelier \\
\hline European call & $5.64 \mathrm{E}-02$ & & $9.67 \mathrm{E}-04$ & $9.47 \mathrm{E}-02$ & $1.91 \mathrm{E}-03$ & & \\
Variance & $5.64 \mathrm{E}-02$ & $1.42 \mathrm{E}-03$ & $5.02 \mathrm{E}-04$ & $9.47 \mathrm{E}-02$ & $1.45 \mathrm{E}-03$ & $2 \%$ & $3 \%$ \\
\hline
\end{tabular}

\section{Table 2}

Error estimate of call by the variance option, $\epsilon=0.01$

\begin{tabular}{|l|l|l|lll|lc|}
\hline & Bachelier & $u^{(\epsilon)}$ & $u_{0}$ & $u_{1}$ & $u_{0}+\epsilon u_{1}$ & Error & $u^{(\epsilon)} /$ Bachelier \\
\hline European call & $5.64 \mathrm{E}-02$ & & $9.67 \mathrm{E}-04$ & $9.47 \mathrm{E}-02$ & $5.70 \mathrm{E}-03$ & & \\
Variance & $5.64 \mathrm{E}-02$ & $4.60 \mathrm{E}-03$ & $5.02 \mathrm{E}-04$ & $9.47 \mathrm{E}-02$ & $5.24 \mathrm{E}-03$ & $14 \%$ & $8 \%$ \\
\hline
\end{tabular}

\section{Table 3}

Error estimate of call by the variance option, $\epsilon=0.05$

\begin{tabular}{|l|l|l|lll|lc|}
\hline & Bachelier & $u^{(\epsilon)}$ & $u_{0}$ & $u_{1}$ & $u_{0}+\epsilon u_{1}$ & Error & $u^{(\epsilon)} /$ Bachelier \\
\hline European call & $5.64 \mathrm{E}-02$ & & $9.67 \mathrm{E}-04$ & $9.47 \mathrm{E}-02$ & $1.04 \mathrm{E}-02$ & & \\
Variance & $5.64 \mathrm{E}-02$ & $7.73 \mathrm{E}-03$ & $5.02 \mathrm{E}-04$ & $9.47 \mathrm{E}-02$ & $9.98 \mathrm{E}-03$ & $29 \%$ & $14 \%$ \\
\hline
\end{tabular}

\section{Table 4}

Error estimate of call by variance option, $\epsilon=0.10$

In these examples, as well as the numerical experiments in Section 6.4, for the European call option, the numbers of time steps and paths for Monte Carlo simulation for $u_{1}$ in (41) are 10,000 and 1,000, respectively. The computation time is 10 seconds, and the $95 \%$ confidence interval for $u_{0}+\epsilon u_{1}$ is $[1.82 \mathrm{E}-03,2.01 \mathrm{E}-03]$, [5.21E-03, 6.19E$03]$, and [9.46E-03, 1.14E-02] for $\epsilon=0.01,0.05$ and 0.10 . Here, we used the following formula for the calculation of the $95 \%$ confidence interval for Monte Carlo simulation of $u_{1} \cdot\left[a-\frac{1.96 b}{\sqrt{M}}, a+\frac{1.96 b}{\sqrt{M}}\right]$, where $a$ and $b$ are the mean and the standard deviation of the samples of the random variable in the Monte Carlo simulation, respectively, and $M$ is the number of the samples in the simulation. The CPU used for this calculation is Intel Core i7-3517U, 1.90GHz. Table 5 illustrates the convergence of $u_{1}$ as the number of time steps increases and the errors from the case in which the number is $1,000,000$. We observe that the error is small enough when the number of time steps is 10,000 . Even with 1,000 time steps, the approximation is useful when quick pricing is necessary while very accurate prices are not required (e.g. when we need to indicate prices for many customers in a short time).

\begin{tabular}{|c|cccc|}
\hline Number of time steps & 1,000 & 10,000 & 100,000 & $1,000,000$ \\
\hline$u_{1}$ & 0.1102 & 0.0947 & 0.0904 & 0.0931 \\
Error & $18.33 \%$ & $1.73 \%$ & $-2.93 \%$ & $0.00 \%$ \\
\hline
\end{tabular}

Table 5

Convergence of $u_{1}$ as \# of time steps increases, $\epsilon=0.10$

The number of time steps for the calculation of $B_{0}$ in (39) is 15 with the double exponential formula for numerical integration (see Takahashi and Mori [17] for instance), and we set $B_{0, x_{1}} \equiv 0$ in computation of integration in (41). Table 6 shows the values of $u_{1}$ when we set $B_{0, x_{1}} \equiv 0$ (without $B_{0, x_{1}}$ ) or the number of time steps in the integration in (40) increases. Here, we assume the value when the number of time steps for $B_{0, x_{1}}$ is 100 as the true value. We observe that the impact of $B_{0, x_{1}}$ on $u_{1}$ is negligible.

\begin{tabular}{|c|ccc|}
\hline Number of time steps for $B_{0, x_{1}}$ & Without $B_{0, x_{1}}$ & 10 & 100 \\
\hline$u_{1}$ & 0.0947 & 0.0943 & 0.0942 \\
Error & $0.55 \%$ & $0.08 \%$ & $0.00 \%$ \\
\hline
\end{tabular}

Table 6

Convergence of $u_{1}$ as \# of time steps for $B_{0, x_{1}}$ increases

When higher accuracy with a narrower confidence interval for the first order approximation is needed, we can set greater numbers of the time steps and the paths for Monte Carlo simulation (e.g. see Section 7.2 where we observe $0.2448(=2.1448-1.9000)$ for $u_{0}+\epsilon u_{1}$ and $[0.2443,0.2453](=[2.1443-1.9000,2.1453-1.9000])$ for its $95 \%$ confidence interval with 10,000 for the number of time steps and 100,000 for the number of paths for Monte Carlo simulation).

\subsection{Comparative statics}

Finally, we observe changes in the hedging cost for the variance option and the European call option in physical settlement when the level of the parameters varies.

Table 7 presents the values of $u_{0}+\epsilon u_{1}$ for the European call options with $K=0.90,1.00$ and 1.10. We observe that $u_{0}+\epsilon u_{1}$ takes the largest value for the at the money strike option. This is because the hedging frequency of the at the money strike option is high, which is caused by the largest derivatives gamma due to the convexity of the payoff.

\begin{tabular}{|l|l|lll|c|c|}
\hline$K$ & Bachelier & $u_{0}$ & $u_{1}$ & $u_{0}+\epsilon u_{1}$ & $\left(u_{0}+\epsilon u_{1}\right) /$ Bachelier & Confidence interval \\
\hline 0.90 & $1.20 \mathrm{E}-01$ & $7.22 \mathrm{E}-04$ & $6.88 \mathrm{E}-02$ & $7.60 \mathrm{E}-03$ & $6 \%$ & {$[6.78 \mathrm{E}-03,8.42 \mathrm{E}-03]$} \\
1.00 & $5.64 \mathrm{E}-02$ & $9.67 \mathrm{E}-04$ & $9.47 \mathrm{E}-02$ & $1.04 \mathrm{E}-02$ & $19 \%$ & {$[9.46 \mathrm{E}-03,1.14 \mathrm{E}-02]$} \\
1.10 & $2.00 \mathrm{E}-02$ & $7.22 \mathrm{E}-04$ & $6.50 \mathrm{E}-02$ & $7.22 \mathrm{E}-03$ & $36 \%$ & {$[6.20 \mathrm{E}-03,8.25 \mathrm{E}-03]$} \\
\hline
\end{tabular}
Table 7

$u_{0}+\epsilon u_{1}$ for call option with different strike levels, $\epsilon=0.10$

In the following, we consider the ATM case $K=1.00$, which is the at the money strike for both options, since it has the largest hedging cost for the European call option. (The results for the ITM and OTM cases are given in Appendix C.) Tables 8-11 present the levels of $u^{(\epsilon)}$ for the variance options when $\eta, \lambda, T$, or $\sigma$ varies, and Tables 12-16 show those of $u_{0}+\epsilon u_{1}$ for the European call option when $\eta, \lambda, T, \sigma$ or $\delta$ changes.

Tables 8 and 9 indicate that the hedging cost increases when the parameter $\eta$ or $\lambda$ increases for the variance option. Tables 12, 13, and 16 also show that the hedging 
cost increases when the parameter $\eta, \lambda$, or $\delta$ increases for the European call option. This implies that when the price slippage of the underlying asset, degree of the risk aversion, or uncertainty on the execution volume increases, the hedging cost becomes large, which agrees with the intuition.

Tables 14 and 15 describe that the ratio of the hedging cost to the Bachelier price decreases in the case of the European call option if $T$ or $\sigma$ increases, while it is unchanged in the case of the variance option as in Tables 10 and 11 . Since the underlying asset price near the maturity is likely to be away from the strike level when $T$ or $\sigma$ is high, which leads to less frequent rehedging in the case of the European call option. Note that the ratio is unchanged in the case of the variance option, since the frequency of rehedging does not depend on the time to maturity or the price level of the underlying asset due to the constant gamma.

- Variance option

\begin{tabular}{|l|l|l|c|}
\hline$\eta$ & Bachelier & $u^{(\epsilon)}$ & $u^{(\epsilon)} /$ Bachelier \\
\hline $1.60 \mathrm{E}-05$ & $2.00 \mathrm{E}-02$ & $3.50 \mathrm{E}-03$ & $18 \%$ \\
$1.60 \mathrm{E}-04$ & $2.00 \mathrm{E}-02$ & $3.87 \mathrm{E}-03$ & $19 \%$ \\
$1.60 \mathrm{E}-03$ & $2.00 \mathrm{E}-02$ & $4.96 \mathrm{E}-03$ & $25 \%$ \\
\hline
\end{tabular}

Table 8 $25 \%$

Comparative statics for variance option for a buyer, $\eta$

\begin{tabular}{|l|l|l|c|}
\hline$\eta$ & Bachelier & $u^{(\epsilon)}$ & $u^{(\epsilon)} /$ Bachelier \\
\hline $1.60 \mathrm{E}-05$ & $2.00 \mathrm{E}-02$ & $3.50 \mathrm{E}-03$ & $18 \%$ \\
$1.60 \mathrm{E}-04$ & $2.00 \mathrm{E}-02$ & $3.87 \mathrm{E}-03$ & $19 \%$ \\
$1.60 \mathrm{E}-03$ & $2.00 \mathrm{E}-02$ & $4.96 \mathrm{E}-03$ & $25 \%$ \\
\hline
\end{tabular}

Comparative statics for variance option for a buyer, $\lambda$

\begin{tabular}{|l|l|l|c|}
\hline$T$ & Bachelier & $u^{(\epsilon)}$ & $u^{(\epsilon)} /$ Bachelier \\
\hline 0.125 & $5.00 \mathrm{E}-03$ & $8.77 \mathrm{E}-04$ & $18 \%$ \\
0.25 & $1.00 \mathrm{E}-02$ & $1.75 \mathrm{E}-03$ & $18 \%$ \\
0.5 & $2.00 \mathrm{E}-02$ & $3.50 \mathrm{E}-03$ & $18 \%$ \\
\hline
\end{tabular}

Table 10

\begin{tabular}{|l|l|l|c|}
\hline$\sigma$ & Bachelier & $u^{(\epsilon)}$ & $u^{(\epsilon)} /$ Bachelier \\
\hline 0.20 & $2.00 \mathrm{E}-02$ & $3.50 \mathrm{E}-03$ & $18 \%$ \\
0.30 & $4.50 \mathrm{E}-02$ & $8.07 \mathrm{E}-03$ & $18 \%$ \\
0.40 & $8.00 \mathrm{E}-02$ & $1.47 \mathrm{E}-02$ & $18 \%$ \\
\hline
\end{tabular}

Table 11

Comparative statics for vari- Comparative statics for variance option for a buyer, $T$

ance option for a buyer, $\sigma$

- European call option

\begin{tabular}{|l|l|lll|c|c|}
\hline$\eta$ & Bachelier & $u_{0}$ & $u_{1}$ & $u_{0}+\epsilon u_{1}$ & $\left(u_{0}+\epsilon u_{1}\right) /$ Bachelier & Confidence interval \\
\hline $1.60 \mathrm{E}-05$ & $5.64 \mathrm{E}-02$ & $9.67 \mathrm{E}-04$ & $9.47 \mathrm{E}-02$ & $1.04 \mathrm{E}-02$ & $19 \%$ & {$[9.46 \mathrm{E}-03,1.14 \mathrm{E}-02]$} \\
$1.60 \mathrm{E}-04$ & $5.64 \mathrm{E}-02$ & $2.78 \mathrm{E}-03$ & $8.11 \mathrm{E}-02$ & $1.09 \mathrm{E}-02$ & $19 \%$ & {$[1.03 \mathrm{E}-02,1.14 \mathrm{E}-02]$} \\
$1.60 \mathrm{E}-03$ & $5.64 \mathrm{E}-02$ & $7.43 \mathrm{E}-03$ & $5.86 \mathrm{E}-02$ & $1.33 \mathrm{E}-02$ & $24 \%$ & {$[1.30 \mathrm{E}-02,1.36 \mathrm{E}-02]$} \\
\hline
\end{tabular}

\section{Table 12}

Comparative statics for call option for a buyer, $\eta$

\begin{tabular}{|l|l|lll|c|c|}
\hline$\lambda$ & Bachelier & $u_{0}$ & $u_{1}$ & $u_{0}+\epsilon u_{1}$ & $\left(u_{0}+\epsilon u_{1}\right) /$ Bachelier & Confidence interval \\
\hline 10 & $5.64 \mathrm{E}-02$ & $9.67 \mathrm{E}-04$ & $9.47 \mathrm{E}-02$ & $1.04 \mathrm{E}-02$ & $19 \%$ & {$[9.46 \mathrm{E}-03,1.14 \mathrm{E}-02]$} \\
100 & $5.64 \mathrm{E}-02$ & $2.25 \mathrm{E}-03$ & $8.91 \mathrm{E}-02$ & $1.12 \mathrm{E}-02$ & $20 \%$ & {$[1.02 \mathrm{E}-02,1.21 \mathrm{E}-02]$} \\
1,000 & $5.64 \mathrm{E}-02$ & $6.44 \mathrm{E}-03$ & $5.89 \mathrm{E}-02$ & $1.23 \mathrm{E}-02$ & $22 \%$ & {$[1.12 \mathrm{E}-02,1.34 \mathrm{E}-02]$} \\
\hline
\end{tabular}

\section{Table 13}

Comparative statics for call option for a buyer, $\lambda$

\begin{tabular}{|l|l|lll|c|c|}
\hline$T$ & Bachelier & $u_{0}$ & $u_{1}$ & $u_{0}+\epsilon u_{1}$ & $\left(u_{0}+\epsilon u_{1}\right) /$ Bachelier & Confidence interval \\
\hline 0.125 & $2.82 \mathrm{E}-02$ & $1.30 \mathrm{E}-03$ & $5.83 \mathrm{E}-02$ & $7.13 \mathrm{E}-03$ & $25 \%$ & {$[5.77 \mathrm{E}-03,8.49 \mathrm{E}-03]$} \\
0.25 & $3.99 \mathrm{E}-02$ & $1.11 \mathrm{E}-03$ & $7.99 \mathrm{E}-02$ & $9.09 \mathrm{E}-03$ & $23 \%$ & {$[7.97 \mathrm{E}-03,1.02 \mathrm{E}-02]$} \\
0.5 & $5.64 \mathrm{E}-02$ & $9.67 \mathrm{E}-04$ & $9.47 \mathrm{E}-02$ & $1.04 \mathrm{E}-02$ & $19 \%$ & {$[9.46 \mathrm{E}-03,1.14 \mathrm{E}-02]$} \\
\hline
\end{tabular}

Table 14

Comparative statics for call option for a buyer, $T$

\begin{tabular}{|l|l|lll|c|c|}
\hline$\sigma$ & Bachelier & $u_{0}$ & $u_{1}$ & $u_{0}+\epsilon u_{1}$ & $\left(u_{0}+\epsilon u_{1}\right) /$ Bachelier & Confidence interval \\
\hline 0.20 & $5.64 \mathrm{E}-02$ & $9.67 \mathrm{E}-04$ & $9.47 \mathrm{E}-02$ & $1.04 \mathrm{E}-02$ & $19 \%$ & {$[9.46 \mathrm{E}-03,1.14 \mathrm{E}-02]$} \\
0.30 & $8.46 \mathrm{E}-02$ & $1.26 \mathrm{E}-03$ & $1.03 \mathrm{E}-01$ & $1.16 \mathrm{E}-02$ & $14 \%$ & {$[1.09 \mathrm{E}-02,1.23 \mathrm{E}-02]$} \\
0.40 & $1.13 \mathrm{E}-01$ & $1.55 \mathrm{E}-03$ & $1.07 \mathrm{E}-01$ & $1.23 \mathrm{E}-02$ & $11 \%$ & {$[1.17 \mathrm{E}-02,1.29 \mathrm{E}-02]$} \\
\hline
\end{tabular}

\section{Table 15}

Comparative statics for call option for a buyer, $\sigma$

\begin{tabular}{|l|l|lll|c|c|}
\hline$\delta$ & Bachelier & $u_{0}$ & $u_{1}$ & $u_{0}+\epsilon u_{1}$ & $\left(u_{0}+\epsilon u_{1}\right) /$ Bachelier & Confidence interval \\
\hline 0.00 & $5.64 \mathrm{E}-02$ & $9.67 \mathrm{E}-04$ & $9.47 \mathrm{E}-02$ & $1.04 \mathrm{E}-02$ & $19 \%$ & {$[9.46 \mathrm{E}-03,1.14 \mathrm{E}-02]$} \\
0.01 & $5.64 \mathrm{E}-02$ & $1.22 \mathrm{E}-03$ & $9.49 \mathrm{E}-02$ & $1.07 \mathrm{E}-02$ & $19 \%$ & {$[9.73 \mathrm{E}-03,1.17 \mathrm{E}-02]$} \\
0.10 & $5.64 \mathrm{E}-02$ & $2.60 \mathrm{E}-02$ & $9.68 \mathrm{E}-02$ & $3.57 \mathrm{E}-02$ & $63 \%$ & {$[3.48 \mathrm{E}-02,3.66 \mathrm{E}-02]$} \\
\hline
\end{tabular}

\section{Table 16}

Comparative statics for call option for a buyer, $\delta$

\section{Comparison with Guéant and $\mathrm{Pu}[7]$}

Guéant and $\mathrm{Pu}$ [7] deal with indifference pricing for a hedger with an exponential utility on the mark-tomarket value of the hedging portfolio at maturity. The model also assumes that the mid price is a Gaussian process with the permanent impact, which is the same as our (1), and the price slippage which is a power function of the speed of order placement. Guéant and $\mathrm{Pu}$ [7] show a single example of a European call option price for a seller when market impact exists by solving a HJB equation numerically by a finite difference method. An advantage of our model over the one in Guéant and $\mathrm{Pu}[7]$ is that the risk neutral price is also obtained by letting $\lambda$ to 0 , since our objective function is separable into the risk neutral part and the risk aversion part which is the expectation of the quadratic variation of the mark-to-market for the portfolio. Moreover, the derivatives prices in our model are computed more easily by the asymptotic expansion.

In the following, we calculate a European option price for a seller using comparable parameters with the numerical example in Guéant and $\mathrm{Pu}$ [7].

\subsection{Parameters}

First, we set the parameters in our model to compare with the numerical example in Guéant and $\mathrm{Pu}$ [7] as follows. $\sigma=0.2150, \delta=0, t=0, T=0.2423, P_{0}=1.00$, $K=1.00, Y_{0}=0, \epsilon=0.01333, \lambda=90, \eta=4.20 * 10^{-5}$, and $b_{2}=0.02284$. In detail, we determine the parameters in the following way.

- $T$ and $\sigma$ : The maturity of 63 days in Guéant and $\mathrm{Pu}$ [7] is converted to 0.2423 years by dividing the number of days by 260 weekdays. $\sigma$ is set so that the standard deviations of both models without the permanent impact match, that is, $\frac{0.6}{45} * \sqrt{63}=\sigma * \sqrt{0.2423}$. In the left hand side, we have normalized the standard deviation of 0.6 by 45 , the initial price of the underlying asset, in Guéant and $\mathrm{Pu}$ [7].

- $\eta$ : In the example of Guéant and $\mathrm{Pu}$ [7], the price slippage when the hedger buys 4 million notional of the underlying asset in one day is 0.10 against 45 of the underlying asset price. Since the speed of order placement $\theta_{t}$ when the hedger buys 4 million notional $\left(0.2\left(=\frac{4}{20}\right)\right.$ units $)$ against 20 million of the option notional in one day ( $\frac{1}{260}$ years) is $\theta_{t}=\frac{0.2}{\frac{1}{260}}=52$, we have $\eta \theta_{t}=\eta * 52=\frac{0.1}{45}$, and hence, $\eta=4.2 * 10^{-5}$.

- $\epsilon$ : In Guéant and $\mathrm{Pu}$ [7], the market impact when the hedger buys 20 million notional of the underlying asset ( 1 unit) is 0.6 against 45 of the underlying asset price. Hence, we have $\epsilon=0.6 / 45=0.0133$.

- $b_{2}$ : The liquidation cost of one unit of the underlying asset at maturity is 1.028 in Guéant and $\mathrm{Pu}$ [7], which is equivalent to $2.28 \%$ of the mid price of the underlying asset. Thus, we set $b_{2}=0.028$.

- $\lambda$ : Expanding the exponential utility used in indifference pricing in Guéant and $\mathrm{Pu}$ [7] up to the second order, we have $-\exp \left(-\alpha\left(\tilde{R}_{T}-\psi\left(P_{T}, Y_{T}\right)\right)\right) \sim-1+$ $\alpha\left(\left(\tilde{R}_{T}-\psi\left(P_{T}, Y_{T}\right)\right)-\frac{1}{2} \alpha\left(\tilde{R}_{T}-\psi\left(P_{T}, Y_{T}\right)\right)^{2}\right)$, where $\tilde{R}_{T}=R_{T}-R_{0}$ and $\alpha=180$. Considering that $\frac{1}{2} \alpha$ corresponds to the risk aversion parameter $\lambda$ in our model, we set $\lambda$ as $\lambda=\frac{1}{2} \alpha=90$. 


\subsection{Result}

With the parameters above, the Bachelier price and the prices without and with market impact in our model, which are rescaled by the underlying asset price in Guéant and $\mathrm{Pu}$ [7], are 1.900, 2.055, and 2.1448, while those in Guéant and $\mathrm{Pu}$ [7] are 1.900, 2.067, and 2.689 , respectively. Here, the $95 \%$ confidence interval for the price with market impact in our model is [2.1443, 2.1453]. The number of the paths for Monte Carlo simulation for $u_{1}$ in (41) is 100,000 , and the other conditions for the calculation are the same as those in Section 6.3. The computation time is 20 minutes. Although the two models show close prices in the case of the models without market impact, the difference is relatively large in the case of the models with market impact, which is due to the different features of the models.

Since the exponential utility rapidly decreases in the negative area, the difference between the two utility functions is large when $\tilde{R}_{T}-\psi$ takes a negative value. When the market impact exists, $\psi$ is large due to the mismatch of the underlying asset position caused by the market impact, and pushes the value of $\tilde{R}_{T}-\psi$ further down, which makes a large difference in values for the two utility functions. Here, we note that $\tilde{R}_{T}$ takes a value close to zero. Note also that if we take $\lambda=1716$ or $\epsilon=0.0941$ meaning that the hedger is more risk averse or degree of the market impact is higher, the prices match between the two models when there exists market impact.

\section{Conclusion}

This paper has presented a stochastic model in continuous time under the existence of market impact and liquidity costs for the underlying asset. This study also provides derivatives pricing with this model through a stochastic control problem, which is a generalized form of the linear-quadratic control and is solved analytically or approximately by an asymptotic expansion.

This method is useful since traders in financial institutions are able to estimate the charge for the liquidity costs and the market impact when they quote derivatives prices. This is particularly important when financial institutions trade derivatives on an underlying asset with low liquidity and there exists market impact on the underlying asset price which is caused by the hedging transactions.

Furthermore, we have provided concrete examples of the charges on the liquidity costs and the market impact for both a variance option in physical settlement, where the charge is solved analytically, and a European call option in physical settlement, where the charge is obtained by the asymptotic expansion. We have also presented comparative static analyses for the parameters' changes in the variance option and the call option in physical settlement.

\section{References}

[1] M.Aliyu (2003): A transformation approach for solving the Hamilton-Jacobi-Bellman equation in $\mathcal{H}^{2}$ deterministic and stochastic optimal control of affine nonlinear systems. Automatica, Vol.39-7, pp.1243-1249.

[2] R. Almgren, N. Chriss (2001). Optimal execution of portfolio transactions. Journal of Risk, 3, 5-40.
[3] R.Beard, G.Saridis, J.Wen (1997): Galerkin approximations of the generalized Hamilton-Jacobi-Bellman equation. Automatica, Vol.33-12, pp.2159-2177.

[4] L.Crespo, J.Sun (2003): Stochastic optimal control via Bellman's principle. Automatica, Vol.39-12, pp.2109-2114.

[5] W.Fleming, R.Rishel (1975): Deterministic and Stochastic Optimal Control. Springer.

[6] J.Gatheral (2010): No-dynamic-arbitrage and market impact. Quantitative finance, 10(7), 749-759.

[7] O.Guéant, J.Pu (2015): Option pricing and hedging with execution costs and market impact. Mathematical Finance, DOI: $10.1111 /$ mafi.12102.

[8] F.B.Hanson (2007): Applied stochastic processes and control for Jump-diffusions: modeling, analysis, and computation (Vol. 13). Siam.

[9] C.Huang, S.Wang, C.Chen, Z.Li (2006): A radial basis collocation method for Hamilton-Jacobi-Bellman equations. Automatica, Vol.42-12, pp.2201-2207.

[10] I.Karatzas, S.Shreve (1988): Brownian Motion and Stochastic Calculus. Springer-Verlag.

[11] T.Li, R.Almgren (2016): Option Hedging with Smooth Market Impact. Market Microstructure and Liquidity Vol. 02, No. 01, 1650002.

[12] F. A. Longstaff (2001): Optimal portfolio choice and the valuation of illiquid securities. Review of financial studies, 14(2), 407-431.

[13] L.C.Rogers, S.Singh (2010): The Cost of Illiquidity and its Effects on Hedging. Mathematical Finance, Vol.20, 597-615.

[14] T.Saito (2015): Self-Financing Strategy Expression in General Shape Limit Order Book with Market Impacts in Continuous Time. International Journal of Financial Engineering, Vol.2-3, 1550034.

[15] W.Schachermayer, J.Teichmann (2008): How close are the option pricing formulas of Bachelier and Black-MertonScholes?. Mathematical Finance, 18(1), 155-170.

[16] A.Takahashi (2015): Asymptotic Expansion Approach in Finance. Large Deviations and Asymptotic Methods in Finance, Vol.110, Ch.13, Springer, pp.345-411.

[17] H.Takahasi, M.Mori (1974): Double exponential formulas for numerical integration. Publications of the Research Institute for Mathematical Sciences, Vol.9-3, pp.721-741.

\section{A Asymptotic expansion for European call pay- off in cash settlement}

In the case of cash settlement of European call option, where $b_{2}=k_{1}, b_{1}=-2 k_{1} g_{x_{1}}\left(T, x_{1}\right), b_{0}=k_{1} g_{x_{1}}^{2}\left(T, x_{1}\right)$ as in (7) and $h\left(x_{1}\right)=\left(x_{1}-K\right)^{+}$, the first order approximation is calculated as follows. Noting that

$$
\begin{gathered}
b_{2}=k_{1}, \\
b_{1}=-2 k_{1} g_{x_{1}}\left(T, x_{1}\right)=-2 k_{1} 1_{\left\{x_{1}>K\right\}}, \\
b_{0}=k_{1} g_{x_{1}}^{2}\left(T, x_{1}\right)=k_{1} 1_{\left\{x_{1}>K\right\}},
\end{gathered}
$$

by (34), we have

$B_{1}\left(t, x_{1}\right)=e^{-\int_{t}^{T} \frac{1}{\eta} B_{2}(s) d s}\left(-2 k_{1} N\left(\frac{x_{1}-K}{\sigma \sqrt{T-t}}\right)\right)$,

$B_{1, x_{1}}\left(t, x_{1}\right)=e^{-\int_{t}^{T} \frac{1}{\eta} B_{2}(s) d s}\left(-2 k_{1} \frac{1}{\sigma \sqrt{T-t}} n\left(\frac{x_{1}-K}{\sigma \sqrt{T-t}}\right)\right)$,

$B_{1, x_{1} x_{1}}\left(t, x_{1}\right)=e^{-\int_{t}^{T} \frac{1}{\eta} B_{2}(s) d s}\left(2 k_{1} \frac{x_{1}-K}{\sigma^{3}(\sqrt{T-t})^{3}} n\left(\frac{x_{1}-K}{\sigma \sqrt{T-t}}\right)\right)$,

where $e^{-\int_{t}^{T} \frac{1}{\eta} B_{2}(s) d s}$ is the one obtained in Section 4.1.

Next, we note that

$$
\mathbf{E}\left[b_{0}\left(x_{1}+\sigma W_{T-t}\right)\right]=-k_{1} N\left(\frac{x_{1}-K}{\sigma \sqrt{T-t}}\right),
$$




$$
\begin{gathered}
\mathbf{E}\left[\sigma^{2} \Gamma\left(s, x_{1}+\sigma W_{s-t}\right) B_{1, x_{1}}\left(s, x_{1}+\sigma W_{s-t}\right)\right] \\
=-2 k_{1} \sigma^{2} e^{-\int_{s}^{T} \frac{1}{\eta} B_{2}(v) d v} \mathbf{E}\left[\Gamma^{2}\left(s, x_{1}+\sigma W_{s-t}\right)\right] \\
=-2 k_{1} \sigma^{2} e^{-\int_{s}^{T} \frac{1}{\eta} B_{2}(v) d v} \\
\quad \cdot \frac{1}{2 \pi \sigma^{2} \sqrt{T-s} \sqrt{T+s-2 t}} e^{-\frac{\left(x_{1}-K\right)^{2}}{\sigma^{2}(T+s-2 t)}} \cdot \quad(\mathrm{A} .5) \\
\mathbf{E}\left[-\frac{1}{4 \eta} B_{1}^{2}\left(s, x_{1}+\sigma W_{s-t}\right)\right] \\
=-\frac{1}{\eta} k_{1}^{2} e^{-2 \int_{s}^{T} \frac{1}{\eta} B_{2}(v) d v} \mathbf{E}\left[N^{2}\left(\frac{x_{1}+\sigma W_{s-t}-K}{\sigma \sqrt{T-s}}\right)\right] \\
=-\frac{1}{\eta} k_{1}^{2} e^{-2 \int_{s}^{T} \frac{1}{\eta} B_{2}(v) d v} \\
{\left[1-\sqrt{\frac{2(s-t)}{\pi(T-t)}} e^{\frac{1}{2}\left(x_{1}-K\right)^{2}\left\{\frac{s-t}{T-t}-\frac{1}{\sigma^{2}(T-s)}\right\}}\right.} \\
\left.\cdot N\left(\sqrt{\frac{T-t}{T+s-2 t}}\left\{\frac{1}{\sigma \sqrt{T-s}}-\frac{s-t}{T-t}\right\}\left(x_{1}-K\right)\right)\right] .
\end{gathered}
$$

Then, in a similar manner as in Example 2, we have

$$
\begin{aligned}
B_{0}\left(t, x_{1}\right) & -k_{1} N\left(\frac{x_{1}-K}{\sigma \sqrt{T-t}}\right) \\
+ & \int_{t}^{T} \frac{1}{2 \pi \sigma^{2} \sqrt{T-s} \sqrt{T+s-2 t}} e^{-\frac{\left(x_{1}-K\right)^{2}}{\sigma^{2}(T+s-2 t)}} \\
& \cdot\left[\tilde{\sigma}^{2} B_{2}(s)+\delta^{2} \lambda \sigma^{2}\left\{\frac{\sigma^{2}(s-t)(T-s)}{T+s-2 t}\right.\right. \\
+ & \left.\left.\left(x_{1}-\frac{2\left(x_{1}-K\right)(s-t)}{T+s-2 t}\right)^{2}\right\}\right] d s \\
+ & \int_{t}^{T}-2 k_{1} \sigma^{2} e^{-\int_{s}^{T} \frac{1}{\eta} B_{2}(v) d v} \\
& \cdot \frac{1}{2 \pi \sigma^{2} \sqrt{T-s} \sqrt{T+s-2 t}} e^{-\frac{\left(x_{1}-K\right)^{2}}{\sigma^{2}(T+s-2 t)}} d s \\
+ & \int_{t}^{T}-\frac{1}{\eta} k_{1}^{2} e^{-2 \int_{s}^{T} \frac{1}{\eta} B_{2}(v) d v} \\
& \cdot\left[1-\sqrt{\left.\frac{2(s-t)}{\pi(T-t)} e^{\frac{1}{2}\left(x_{1}-K\right)^{2}\left\{\frac{s-t}{T-t}-\frac{1}{\sigma^{2}(T-s)}\right.}\right\}}\right. \\
& \cdot N\left(\sqrt{\frac{T-t}{T+s-2 t}}\left\{\frac{1}{\sigma \sqrt{T-s}}-\frac{s-t}{T-t}\right\}\right. \\
& {\left.\left[x_{1}-K\right)\right) d s . }
\end{aligned}
$$

$B_{0, x_{1}}$ is obtained by taking partial derivative of this with respect to $x_{1} ; u^{(0)}$ and $u^{(1,0)}$ are calculated by (29) and (37).

\section{B Asymptotic expansion for variance payoff in physical settlement}

In this appendix, we provide the expression of $u^{(0)}$ in Section 4.1 and $u^{(1,0)}$ in Section 4.2 for the variance payoff $h\left(x_{1}\right)=\frac{1}{2} \gamma x_{1}^{2}+c_{1} x_{1}+c_{0}$ in Case 1 in the case of physical settlement, where $b_{1}=b_{0}=0$. Here, $\delta$ is not necessarily 0 as in Example 1 in Section 5.

First, we note that $B_{1}=0$, since $b_{1}=0$ in (34).
Since $\Gamma\left(t, x_{1}\right)=\gamma$, we have by $(35)$,

$B_{0}\left(t, x_{1}\right)$

$$
\begin{aligned}
& =\gamma^{2} \tilde{\sigma}^{2} \int_{t}^{T} B_{2}(s) d s+\gamma^{2} \delta^{2} \lambda \sigma^{2} \int_{t}^{T} \mathbf{E}\left[\left(x_{1}+\sigma W_{s-t}\right)^{2}\right] d s \\
& =\gamma^{2} \tilde{\sigma}^{2} \int_{t}^{T} B_{2}(s) d s+\gamma^{2} \delta^{2} \lambda \sigma^{2}\left\{x_{1}^{2}(T-t)+\frac{1}{2} \sigma^{2}(T-t)^{2}\right\}
\end{aligned}
$$

where $B_{2}(t)$ is given as (33) in Section 4.1.

Note also that

$$
\int_{t}^{T} B_{2}(s) d s=\left\{\begin{array}{l}
\eta \log \frac{\cosh \left(-\frac{1}{2} \log \frac{1-h_{0}}{1+h_{0}}+\sqrt{\frac{\lambda \sigma^{2}}{\eta}}(T-t)\right)}{\cosh \left(-\frac{1}{2} \log \frac{1-h_{0}}{1+h_{0}}\right)}, \\
\text { if } 0<b_{2}<\sqrt{\lambda \eta \sigma^{2}}, \\
\sqrt{\lambda \eta \sigma^{2}}(T-t), \text { if } b_{2}=\sqrt{\lambda \eta \sigma^{2}}, \\
\eta \log \frac{\sinh \left(-\frac{1}{2} \log \frac{h_{0}-1}{h_{0}+1}+\sqrt{\frac{\lambda \sigma^{2}}{\eta}}(T-t)\right)}{\sinh \left(-\frac{1}{2} \log \frac{h_{0}-1}{h_{0}+1}\right)}, \\
\text { if } \sqrt{\lambda \eta \sigma^{2}}<b_{2},
\end{array}\right.
$$$$
\text { where } h_{0}=\frac{b_{2}}{\sqrt{\lambda \eta \sigma^{2}}} \text {. }
$$

Hence

$$
B_{0, x_{1}}\left(t, x_{1}\right)=2 \gamma^{2} \delta^{2} \lambda \sigma^{2} x_{1}(T-t),
$$

and by (37), we have

$$
\begin{aligned}
& u^{(1,0)}\left(t, x_{1}, x_{2}\right) \\
& =\mathbf{E}^{\left(t, x_{1}, x_{2}\right)}\left[\int_{t}^{T}-\frac{1}{\eta} B_{2}(s) X_{2, s}\right. \\
& \left.\qquad \cdot\left\{2 \gamma B_{2}(s) X_{2, s}+2 \gamma^{2} \delta^{2} \lambda \sigma^{2} X_{1, s}(T-s)-X_{2, s}\right\} d s\right], \\
& \text { where } \\
& \qquad d X_{1, s}=\sigma d W_{1, s} \\
& d X_{2, s}=\sigma \gamma\left(d W_{1, s}+\delta d W_{2, s}\right)-\frac{1}{\eta} B_{2}(s) X_{2}(s) d s .
\end{aligned}
$$

\section{Comparative statics for the European call op- tion with $K=0.90$ and 1.10}

C.1 In the money payoff, $K=0.90$

This section provides results of the comparative statics for the European call option in Section 6 with $K=0.90$ and 1.10 , which are the in the money and the out of the money case, respectively. We keep the other parameters the same as the ones in Section 6.

\begin{tabular}{|l|l|lll|c|c|}
\hline$\eta$ & Bachelier & $u_{0}$ & $u_{1}$ & $u_{0}+\epsilon u_{1}$ & $\left(u_{0}+\epsilon u_{1}\right) /$ Bachelier & Confidence interval \\
\hline $1.60 \mathrm{E}-05$ & $1.20 \mathrm{E}-01$ & $7.22 \mathrm{E}-04$ & $6.88 \mathrm{E}-02$ & $7.60 \mathrm{E}-03$ & $6 \%$ & {$[6.78 \mathrm{E}-03,8.42 \mathrm{E}-03]$} \\
$1.60 \mathrm{E}-04$ & $1.20 \mathrm{E}-01$ & $2.06 \mathrm{E}-03$ & $6.23 \mathrm{E}-02$ & $8.29 \mathrm{E}-03$ & $7 \%$ & {$[7.80 \mathrm{E}-03,8.78 \mathrm{E}-03]$} \\
$1.60 \mathrm{E}-03$ & $1.20 \mathrm{E}-01$ & $5.47 \mathrm{E}-03$ & $4.93 \mathrm{E}-02$ & $1.04 \mathrm{E}-02$ & $9 \%$ & {$[1.01 \mathrm{E}-02,1.07 \mathrm{E}-02]$} \\
\hline
\end{tabular}

Table C.1

Comparative statics for European call option for a buyer, when $\eta$ changes, $K=0.90$

\begin{tabular}{|l|l|lll|c|c|}
\hline$\lambda$ & Bachelier & $u_{0}$ & $u_{1}$ & $u_{0}+\epsilon u_{1}$ & $\left(u_{0}+\epsilon u_{1}\right) /$ Bachelier & Confidence interval \\
\hline 10 & $1.20 \mathrm{E}-01$ & $7.22 \mathrm{E}-04$ & $6.88 \mathrm{E}-02$ & $7.60 \mathrm{E}-03$ & $6 \%$ & {$[6.78 \mathrm{E}-03,8.42 \mathrm{E}-03]$} \\
100 & $1.20 \mathrm{E}-01$ & $1.65 \mathrm{E}-03$ & $6.41 \mathrm{E}-02$ & $8.07 \mathrm{E}-03$ & $7 \%$ & {$[7.27 \mathrm{E}-03,8.86 \mathrm{E}-03]$} \\
1,000 & $1.20 \mathrm{E}-01$ & $4.70 \mathrm{E}-03$ & $4.46 \mathrm{E}-02$ & $9.16 \mathrm{E}-03$ & $8 \%$ & {$[8.31 \mathrm{E}-03,1.00 \mathrm{E}-02]$} \\
\hline
\end{tabular}

Table C.2

Comparative statics for European call option for a buyer, when $\lambda$ changes, $K=0.90$ 


\begin{tabular}{|l|l|lll|c|c|}
\hline$T$ & Bachelier & $u_{0}$ & $u_{1}$ & $u_{0}+\epsilon u_{1}$ & $\left(u_{0}+\epsilon u_{1}\right) /$ Bachelier & Confidence interval \\
\hline 0.125 & $1.03 \mathrm{E}-01$ & $4.27 \mathrm{E}-04$ & $1.79 \mathrm{E}-02$ & $2.22 \mathrm{E}-03$ & $2 \%$ & {$[1.22 \mathrm{E}-03,3.22 \mathrm{E}-03]$} \\
0.25 & $1.08 \mathrm{E}-01$ & $6.24 \mathrm{E}-04$ & $4.21 \mathrm{E}-02$ & $4.84 \mathrm{E}-03$ & $4 \%$ & {$[3.83 \mathrm{E}-03,5.84 \mathrm{E}-03]$} \\
0.5 & $1.20 \mathrm{E}-01$ & $7.22 \mathrm{E}-04$ & $6.88 \mathrm{E}-02$ & $7.60 \mathrm{E}-03$ & $6 \%$ & {$[6.78 \mathrm{E}-03,8.42 \mathrm{E}-03]$} \\
\hline
\end{tabular}

\section{Table C.3}

Comparative statics for European call option for a buyer, when $T$ changes, $K=0.90$

\begin{tabular}{|l|l|lll|c|c|}
\hline$\sigma$ & Bachelier & $u_{0}$ & $u_{1}$ & $u_{0}+\epsilon u_{1}$ & $\left(u_{0}+\epsilon u_{1}\right) /$ Bachelier & Confidence interval \\
\hline 0.20 & $1.20 \mathrm{E}-01$ & $7.22 \mathrm{E}-04$ & $6.88 \mathrm{E}-02$ & $7.60 \mathrm{E}-03$ & $6 \%$ & {$[6.78 \mathrm{E}-03,8.42 \mathrm{E}-03]$} \\
0.30 & $1.44 \mathrm{E}-01$ & $1.10 \mathrm{E}-03$ & $9.02 \mathrm{E}-02$ & $1.01 \mathrm{E}-02$ & $7 \%$ & {$[9.60 \mathrm{E}-03,1.06 \mathrm{E}-02]$} \\
0.40 & $1.70 \mathrm{E}-01$ & $1.44 \mathrm{E}-03$ & $9.83 \mathrm{E}-02$ & $1.13 \mathrm{E}-02$ & $7 \%$ & {$[1.07 \mathrm{E}-02,1.18 \mathrm{E}-02]$} \\
\hline
\end{tabular}

\section{Table C.4}

Comparative statics for European call option for a buyer, when $\sigma$ changes, $K=0.90$

\begin{tabular}{|l|l|lll|c|c|}
\hline$\delta$ & Bachelier & $u_{0}$ & $u_{1}$ & $u_{0}+\epsilon u_{1}$ & $\left(u_{0}+\epsilon u_{1}\right) /$ Bachelier & Confidence interval \\
\hline 0.00 & $1.20 \mathrm{E}-01$ & $7.22 \mathrm{E}-04$ & $6.88 \mathrm{E}-02$ & $7.60 \mathrm{E}-03$ & $6 \%$ & {$[6.78 \mathrm{E}-03,8.42 \mathrm{E}-03]$} \\
0.01 & $1.20 \mathrm{E}-01$ & $8.78 \mathrm{E}-04$ & $6.87 \mathrm{E}-02$ & $7.75 \mathrm{E}-03$ & $6 \%$ & {$[6.93 \mathrm{E}-03,8.58 \mathrm{E}-03]$} \\
0.10 & $1.20 \mathrm{E}-01$ & $1.64 \mathrm{E}-02$ & $6.90 \mathrm{E}-02$ & $2.33 \mathrm{E}-02$ & $19 \%$ & {$[2.24 \mathrm{E}-02,2.42 \mathrm{E}-02]$} \\
\hline
\end{tabular}

\section{Table C.5}

Comparative statics for European call option for a buyer, when $\delta$ changes, $K=0.90$

\section{C.2 Out of the money payoff, $K=1.10$}

\begin{tabular}{|l|l|lll|c|c|}
\hline$\eta$ & Bachelier & $u_{0}$ & $u_{1}$ & $u_{0}+\epsilon u_{1}$ & $\left(u_{0}+\epsilon u_{1}\right) /$ Bachelier & Confidence interval \\
\hline $1.60 \mathrm{E}-05$ & $2.00 \mathrm{E}-02$ & $7.22 \mathrm{E}-04$ & $6.50 \mathrm{E}-02$ & $7.22 \mathrm{E}-03$ & $36 \%$ & {$[6.20 \mathrm{E}-03,8.25 \mathrm{E}-03]$} \\
$1.60 \mathrm{E}-04$ & $2.00 \mathrm{E}-02$ & $2.06 \mathrm{E}-03$ & $6.23 \mathrm{E}-02$ & $8.29 \mathrm{E}-03$ & $42 \%$ & {$[7.77 \mathrm{E}-03,8.81 \mathrm{E}-03]$} \\
$1.60 \mathrm{E}-03$ & $2.00 \mathrm{E}-02$ & $5.47 \mathrm{E}-03$ & $4.86 \mathrm{E}-02$ & $1.03 \mathrm{E}-02$ & $52 \%$ & {$[1.00 \mathrm{E}-02,1.06 \mathrm{E}-02]$} \\
\hline
\end{tabular}

\section{Table C.6}

Comparative statics for European call option for a buyer, when $\eta$ changes, $K=1.10$

\begin{tabular}{|l|l|lll|c|c|}
\hline$\lambda$ & Bachelier & $u_{0}$ & $u_{1}$ & $u_{0}+\epsilon u_{1}$ & $\left(u_{0}+\epsilon u_{1}\right) /$ Bachelier & Confidence interval \\
\hline 10 & $2.00 \mathrm{E}-02$ & $7.22 \mathrm{E}-04$ & $6.50 \mathrm{E}-02$ & $7.22 \mathrm{E}-03$ & $36 \%$ & {$[6.20 \mathrm{E}-03,8.25 \mathrm{E}-03]$} \\
100 & $2.00 \mathrm{E}-02$ & $1.65 \mathrm{E}-03$ & $6.01 \mathrm{E}-02$ & $7.66 \mathrm{E}-03$ & $38 \%$ & {$[6.65 \mathrm{E}-03,8.67 \mathrm{E}-03]$} \\
1,000 & $2.00 \mathrm{E}-02$ & $4.70 \mathrm{E}-03$ & $4.01 \mathrm{E}-02$ & $8.71 \mathrm{E}-03$ & $44 \%$ & {$[7.64 \mathrm{E}-03,9.78 \mathrm{E}-03]$} \\
\hline
\end{tabular}

\section{Table C.7}

Comparative statics for European call option for a buyer, when $\lambda$ changes, $K=1.10$

\begin{tabular}{|l|l|lll|c|c|}
\hline$T$ & Bachelier & $u_{0}$ & $u_{1}$ & $u_{0}+\epsilon u_{1}$ & $\left(u_{0}+\epsilon u_{1}\right) /$ Bachelier & Confidence interval \\
\hline 0.125 & $2.51 \mathrm{E}-03$ & $4.27 \mathrm{E}-04$ & $1.88 \mathrm{E}-02$ & $2.31 \mathrm{E}-03$ & $92 \%$ & {$[1.44 \mathrm{E}-03,3.17 \mathrm{E}-03]$} \\
0.25 & $8.33 \mathrm{E}-03$ & $6.24 \mathrm{E}-04$ & $3.95 \mathrm{E}-02$ & $4.57 \mathrm{E}-03$ & $55 \%$ & {$[3.54 \mathrm{E}-03,5.60 \mathrm{E}-03]$} \\
0.5 & $2.00 \mathrm{E}-02$ & $7.22 \mathrm{E}-04$ & $6.50 \mathrm{E}-02$ & $7.22 \mathrm{E}-03$ & $36 \%$ & {$[6.20 \mathrm{E}-03,8.25 \mathrm{E}-03]$} \\
\hline
\end{tabular}

Table C.8

Comparative statics for European call option for a buyer, when $T$ changes, $K=1.10$

\begin{tabular}{|l|l|lll|c|c|}
\hline$\sigma$ & Bachelier & $u_{0}$ & $u_{1}$ & $u_{0}+\epsilon u_{1}$ & $\left(u_{0}+\epsilon u_{1}\right) /$ Bachelier & Confidence interval \\
\hline 0.20 & $2.00 \mathrm{E}-02$ & $7.22 \mathrm{E}-04$ & $6.50 \mathrm{E}-02$ & $7.22 \mathrm{E}-03$ & $36 \%$ & {$[6.20 \mathrm{E}-03,8.25 \mathrm{E}-03]$} \\
0.30 & $4.39 \mathrm{E}-02$ & $1.10 \mathrm{E}-03$ & $8.66 \mathrm{E}-02$ & $9.76 \mathrm{E}-03$ & $22 \%$ & {$[8.99 \mathrm{E}-03,1.05 \mathrm{E}-02]$} \\
0.40 & $6.98 \mathrm{E}-02$ & $1.44 \mathrm{E}-03$ & $9.79 \mathrm{E}-02$ & $1.12 \mathrm{E}-02$ & $16 \%$ & {$[1.07 \mathrm{E}-02,1.18 \mathrm{E}-02]$} \\
\hline
\end{tabular}

Table C.9

Comparative statics for European call option for a buyer, when $\sigma$ changes, $K=1.10$

\begin{tabular}{|l|l|lll|c|c|}
\hline$\delta$ & Bachelier & $u_{0}$ & $u_{1}$ & $u_{0}+\epsilon u_{1}$ & $\left(u_{0}+\epsilon u_{1}\right) /$ Bachelier & Confidence interval \\
\hline 0.00 & $2.00 \mathrm{E}-02$ & $7.22 \mathrm{E}-04$ & $6.50 \mathrm{E}-02$ & $7.22 \mathrm{E}-03$ & $36 \%$ & {$[6.20 \mathrm{E}-03,8.25 \mathrm{E}-03]$} \\
0.01 & $2.00 \mathrm{E}-02$ & $9.33 \mathrm{E}-04$ & $6.51 \mathrm{E}-02$ & $7.44 \mathrm{E}-03$ & $37 \%$ & {$[6.42 \mathrm{E}-03,8.47 \mathrm{E}-03]$} \\
0.10 & $2.00 \mathrm{E}-02$ & $2.18 \mathrm{E}-02$ & $6.64 \mathrm{E}-02$ & $2.85 \mathrm{E}-02$ & $143 \%$ & {$[2.74 \mathrm{E}-02,2.95 \mathrm{E}-02]$} \\
\hline
\end{tabular}

\section{Table C.10}

Comparative statics for European call option for a buyer, when $\delta$ changes, $K=1.10$

\section{Calculation of the second order expansion}

The second order approximation (expansion) $\epsilon^{\prime 2} \frac{1}{2} u^{(2,0)}$ is calculated through taking the second order derivatives with respect to $\epsilon^{\prime}$ for the original PDE in (18) and substituting $\epsilon^{\prime}=0$, which is given by substituting $\epsilon^{\prime}=0$ into $v^{(\epsilon)}$ in $(23)$. In this way, we obtain the PDE for the second order term as

$$
\begin{gathered}
0=u_{t}^{(2,0)}+\frac{1}{2} \sigma^{2} u_{x_{1}, x_{1}}^{(2,0)}+\sigma^{2} \Gamma\left(t, x_{1}\right) u_{x_{1}, x_{2}}^{(2,0)} \\
+\frac{1}{2} \tilde{\sigma}^{2} \Gamma\left(t, x_{1}\right)^{2} u_{x_{2}, x_{2}}^{(2,0)}-\frac{1}{2 \eta} u_{x_{2}}^{(0)} u_{x_{2}}^{(2,0)}+h^{(0)}\left(t, x_{1}, x_{2}\right), \\
u^{(2,0)}\left(T, x_{1}, x_{2}\right)=0,
\end{gathered}
$$

where

$$
\begin{gathered}
h^{(0)}\left(t, x_{1}, x_{2}\right)=-\frac{1}{4 \eta}\left(\Gamma\left(t, x_{1}\right) u_{x_{2}}^{(0)}+u_{x_{2}}^{(1,0)}+u_{x_{1}}^{(0)}-x_{2}\right)^{2} \\
-\frac{1}{2 \eta} u_{x_{2}}^{(0)}\left(\Gamma\left(t, x_{1}\right) u_{x_{2}}^{(1,0)}+u_{x_{1}}^{(1,0)}\right) .
\end{gathered}
$$

The Feynman-Kac representation of $u^{(2,0)}$ is

$$
u^{(2,0)}(t, \mathbf{x})=\mathbf{E}^{(t, \mathbf{x})}\left[\int_{t}^{T} h^{(0)}\left(s, \mathbf{X}_{s}\right) d s\right]
$$

where $\mathbf{X}_{t}=\left(X_{1, t}, X_{2, t}\right),\left(\begin{array}{l}d X_{1, t} \\ d X_{2, t}\end{array}\right)=\alpha^{(0)}\left(t, X_{1, t}, X_{2, t}\right) d t+$

$\sigma\left(t, X_{1, t}\right)\left(\begin{array}{l}d W_{1, t} \\ d W_{2, t}\end{array}\right), \alpha^{(0)}\left(t, x_{1}, x_{2}\right)=\left(\begin{array}{c}0 \\ -\frac{1}{2 \eta}\end{array}\right)$,

and $\sigma\left(t, x_{1}\right)=\left(\begin{array}{cc}\sigma & 0 \\ \sigma \Gamma\left(t, x_{1}\right) & \delta \sigma \Gamma\left(t, x_{1}\right)\end{array}\right)$. As in the cal-

culation of the first order term, the second order term can be computed by Monte Carlo simulation via this Feynman-Kac representation. Since the representation of the second order term includes the zeroth order term as well as the first order terms differentiated by $x_{1}$ and $x_{2}$, the the Monte Carlo simulation is nested, which is possible in principle but requires high computational burden. In detail, $u_{x_{1}}^{(1,0)}$ and $u_{x_{2}}^{(1,0)}$ in (D.1) are obtained as follows: By using (discretized) processes $X_{1, s}, X_{2, s}$, $\frac{\partial X_{1, s}}{\partial x_{1}}, \frac{\partial X_{1, s}}{\partial x_{2}}, \frac{\partial X_{2, s}}{\partial x_{1}}$, and $\frac{\partial X_{2, s}}{\partial x_{2}}$ defined as $X_{1, s}=$ $x_{1}+\int_{t}^{s} \sigma d W_{1, v}, X_{2, s}=x_{2}+\int_{t}^{s} \sigma \Gamma\left(s, X_{1, v}\right)\left(d W_{1, v}+\right.$ $\left.\delta d W_{2, v}\right)-\int_{t}^{s} \frac{1}{2 \eta} u_{x_{2}}^{(0)}\left(s, X_{1, v}, X_{2, v}\right) d v, \quad \frac{\partial X_{1, s}}{\partial x_{1}}=1$, $\frac{\partial X_{1, s}}{\partial x_{2}}=0, \frac{\partial X_{2, s}}{\partial x_{1}}=\int_{t}^{s} \sigma \Gamma_{x_{1}}\left(v, X_{1, v}\right)\left(d W_{1, v}+\delta d W_{2, v}\right)-$ $\int_{t}^{s} \frac{1}{2 \eta}\left(B_{1, x_{1}}\left(v, X_{1, v}\right)\right)+2 B_{2}(v) \frac{\partial X_{2, v}}{\partial x_{1}} d v \quad, \quad \frac{\partial X_{2, s}}{\partial x_{2}}=$ $1-\int_{t}^{s} \frac{1}{\eta} B_{2}(v) \frac{\partial X_{2, v}}{\partial x_{2}} d v$, we evaluate $u_{x_{i}}^{(1,0)}\left(t, x_{1}, x_{2}\right)$, $i=1,2$ by the right hand sides of the following equations through Monte Carlo simulations:

$$
\begin{aligned}
& u_{x_{1}}^{(1,0)}\left(t, x_{1}, x_{2}\right)=\mathbf{E}\left[\int_{t}^{T}-\frac{1}{2 \eta}\left\{B_{1, x_{1}}\left(x, X_{1, s}\right)+2 B_{2}(s) \frac{\partial X_{2, s}}{\partial x_{1}}\right\}\right. \\
& \times\left\{\Gamma\left(x, X_{1, s}\right)\left(2 B_{2}(s) X_{2, s}+B_{1}\left(s, X_{1, s}\right)\right)+B_{1, x_{1}}\left(s, X_{1, s}\right) X_{2, s}\right. \\
& \left.+B_{0, x_{1}}\left(s, X_{1, s}\right)-X_{2, s}\right\} d s+\int_{t}^{T}-\frac{1}{2 \eta}\left(2 B_{2}(s) X_{2, s}+B_{1}\left(s, X_{1, s}\right)\right) \\
& \quad \times\left\{\Gamma_{x_{1}}\left(s, X_{1, s}\right)\left(2 B_{2}(s) X_{2, s}+B_{1}\left(s, X_{1, s}\right)\right)\right. \\
& +\Gamma\left(s, X_{1, s}\right)\left(B_{1, x_{1}}\left(s, X_{1, s}\right)+2 B_{2}(s) \frac{\partial X_{2, s}}{\partial x_{1}}\right) \\
& +\left(B_{1, x_{1} x_{1}}\left(s, X_{1, s}\right) X_{2, s}+B_{0, x_{1} x_{1}}\left(s, X_{1, s}\right)\right) \\
& \left.\left.+B_{1, x_{1}}\left(s, X_{1, s}\right) \frac{\partial X_{2, s}}{\partial x_{1}}-\frac{\partial X_{2, s}}{\partial x_{1}}\right\} d s\right]
\end{aligned}
$$




$$
\begin{gathered}
u_{x_{2}}^{(1,0)}\left(t, x_{1}, x_{2}\right)=\mathbf{E}\left[\int_{t}^{T}-\frac{1}{\eta} B_{2}(s) \frac{\partial X_{2, s}}{\partial x_{2}}\right. \\
\times\left\{\Gamma\left(x, X_{1, s}\right)\left(2 B_{2}(s) X_{2, s}+B_{1}\left(s, X_{1, s}\right)\right)+B_{1, x_{1}}\left(s, X_{1, s}\right) X_{2, s}\right. \\
\left.+B_{0, x_{1}}\left(s, X_{1, s}\right)-X_{2, s}\right\} d s+\int_{t}^{T}-\frac{1}{2 \eta}\left(2 B_{2}(s) X_{2, s}+B_{1}\left(s, X_{1, s}\right)\right) \\
\left.\times\left\{\Gamma\left(s, X_{1, s}\right)\left(2 B_{2}(s) \frac{\partial X_{2, s}}{\partial x_{2}}\right)+B_{1, x_{1}}\left(s, X_{1, s}\right) \frac{\partial X_{2, s}}{\partial x_{2}}-\frac{\partial X_{2, s}}{\partial x_{2}}\right\} d s\right] .
\end{gathered}
$$

More specifically, for the variance option with $\delta$ not necessarily being 0 in Appendix B in the on-line version which includes the case in Example 1, and the European call option in Example 2, we have the following expressions.

- Variance option: $\frac{\partial X_{1, s}}{\partial x_{1}}=1, \frac{\partial X_{1, s}}{\partial x_{2}}=0, \frac{\partial X_{2, s}}{\partial x_{1}}=0$ , $\frac{\partial X_{2, s}}{\partial x_{2}}=1-\int_{t}^{s} \frac{1}{\eta} B_{2}(v) \frac{\partial X_{2, v}}{\partial x_{2}} d v, u_{x_{1}}^{(1,0)}\left(t, x_{1}, x_{2}\right)=$ $\mathbf{E}\left[\int_{t}^{T}-\frac{1}{\eta} B_{2}(s) X_{2, s}\left(2 \gamma^{2} \delta^{2} \lambda \sigma^{2}(T-s)\right) d s\right], u_{x_{2}}^{(1,0)}\left(t, x_{1}, x_{2}\right)=$
$\mathbf{E}\left[\int_{t}^{T}-\frac{1}{\eta} B_{2}(s) \frac{\partial X_{2, s}}{\partial x_{2}}\left\{2 \gamma B_{2}(s) X_{2, s}+2 \gamma^{2} \delta^{2} \lambda \sigma^{2} X_{1, s}(T-\right.\right.$
$\left.\left.s)-X_{2, s}\right\} d s+\int_{t}^{T}-\frac{1}{\eta} B_{2}(s) X_{2, s}\left\{2 \gamma B_{2}(s) \frac{\partial X_{2, s}}{\partial x_{2}}-\frac{\partial X_{2, s}}{\partial x_{2}}\right\} d s\right]$.

- European Call: $X_{1, s}=x_{1}+\sigma \int_{t}^{T} d W_{1, v}, X_{2, s}=x_{2}+$ $\int_{t}^{s} \sigma \Gamma\left(v, X_{1, v}\right)\left(d W_{1, v}+\delta d W_{2, v}\right)-\int_{s}^{t} \frac{1}{\eta} B_{2}(v) X_{2, v} d v$, $\frac{\partial X_{1, s}}{\partial x_{1}}=1, \frac{\partial X_{1, s}}{\partial x_{2}}=0, \frac{\partial X_{2, s}}{\partial x_{1}}=\int_{t}^{s} \sigma \Gamma_{x_{1}}\left(v, X_{1, v}\right)\left(d W_{1, v}+\right.$ $\left.\delta d W_{2, v}\right)-\int_{s}^{t} \frac{1}{\eta} B_{2}(v) \frac{\partial X_{2, v}}{\partial x_{1}} d v, \frac{\partial X_{2, s}}{\partial x_{2}}=1-\int_{s}^{t} \frac{1}{\eta} B_{2}(v) \frac{\partial X_{2, v}}{\partial x_{2}} d v$, $u_{x_{1}}^{(1,0)}=\mathbf{E}^{\left(t, x_{1}, x_{2}\right)}\left[\int_{t}^{T}-\frac{1}{\eta} B_{2}(s) \frac{\partial X_{2, s}}{\partial x_{1}}\left\{\Gamma\left(s, X_{1, s}\right) 2 B_{2}(s) X_{2, s}+\right.\right.$ $\left.B_{0, x_{1}}\left(s, X_{1, s}\right)-X_{2, s}\right\} d s+\int_{t}^{T}-\frac{1}{\eta} B_{2}(s) X_{2, s}$ $\times\left\{\Gamma_{x_{1}}\left(s, X_{1, s}\right) 2 B_{2}(s) X_{2, s}+\Gamma\left(s, X_{1, s}\right) 2 B_{2}(s) \frac{\partial X_{2, s}}{\partial x_{1}}\right.$ $\left.\left.+B_{0, x_{1} x_{1}}\left(s, X_{1, s}\right)-\frac{\partial X_{2, s}}{\partial x_{1}}\right\} d s\right]$, $u_{x_{2}}^{(1,0)}=\mathbf{E}^{\left(t, x_{1}, x_{2}\right)}\left[\int_{t}^{T}-\frac{1}{\eta} B_{2}(s) \frac{\partial X_{2, s}}{\partial x_{2}}\left\{\Gamma\left(s, X_{1, s}\right) 2 B_{2}(s) X_{2, s}+\right.\right.$ $\left.B_{0, x_{1}}\left(s, X_{1, s}\right)-X_{2, s}\right\} d s$ $\left.+\int_{t}^{T}-\frac{1}{\eta} B_{2}(s) X_{2, s}\left\{\Gamma\left(s, X_{1, s}\right) 2 B_{2}(s) \frac{\partial X_{2, s}}{\partial x_{2}}-\frac{\partial X_{2, s}}{\partial x_{2}}\right\} d s\right]$. 